\title{
The Riemann boundary value problem related to the time-harmonic Maxwell equations
}

\author{
Pei Yang ${ }^{1,2}$, Liping Wang ${ }^{2 *}$ and Zuoliang $\mathrm{Xu}^{1 *}$
}

"Correspondence: wlpxjj@163.com; xuz|@ruc.edu.cn

'School of Mathematics, Renmin University of China, Beijing, 100872 P.R. China

${ }^{2}$ School of Mathematical Sciences, Hebei Normal University, Shijiazhuang, Hebei Province, 050024, P.R. China

\section{Springer}

\begin{abstract}
In this paper, we first give the definition of Teodorescu operator related to the $\mathcal{N}$ matrix operator and discuss a series of properties of this operator, such as uniform boundedness, Hölder continuity and so on. Then we propose the Riemann boundary value problem related to the $\mathcal{N}$ matrix operator. Finally, using the intimate relationship of the corresponding Cauchy-type integral between the $\mathcal{N}$ matrix operator and the time-harmonic Maxwell equations, we investigate the Riemann boundary value problem related to the time-harmonic Maxwell equations and obtain the integral representation of the solution.
\end{abstract}

Keywords: Quaternion analysis; Riemann boundary value problem; Time-harmonic Maxwell equations; Teodorescu operator; $\mathcal{N}$ matrix operator

\section{Introduction}

The boundary value problem for partial differential equations is a very meaningful research subject which has important applications in physics, chemistry, financial mathematics and many other fields. Teodorescu operator is a generalized solution of the inhomogeneous Dirac equation and it has been widely used in solving the boundary value problem of partial differential equations. Therefore many experts and scholars studied the properties of the Teodorescu operator and corresponding boundary value problem, for example, Vekua N [1] first discussed some properties of the Teodorescu operator on the plane and its application in the shell theory and gas dynamics. Hile GN [2] and Gilbert $\mathrm{RP}$ et al. [3] studied some properties of the Teodorescu operator in n-dimensional Euclidean space and higher-dimensional complex spaces. Du JY, Yang PW, Qiao YY, Taira K and Wang LP et al. studied some properties and boundary value problems associated with the Teodorescu operator in quaternion analysis and Clifford analysis (see [4-16]).

Quaternion analysis is an important branch of modern analysis, which studies the functions defined in the domain of $\mathrm{n}$-dimensional Euclidean space with values in quaternion spaces. It is an important tool for the solution of boundary value problems of highdimensional partial differential equations, including Maxwell equations. The Maxwell equations are a set of partial differential equations describing the relationship between

(c) The Author(s) 2021. This article is licensed under a Creative Commons Attribution 4.0 International License, which permits use sharing, adaptation, distribution and reproduction in any medium or format, as long as you give appropriate credit to the original author(s) and the source, provide a link to the Creative Commons licence, and indicate if changes were made. The images or other third party material in this article are included in the article's Creative Commons licence, unless indicated otherwise in a credit line to the material. If material is not included in the article's Creative Commons licence and your intended use is not permitted by statutory regulation or exceeds the permitted use, you will need to obtain permission directly from the copyright holder. To view a copy of this licence, visit http://creativecommons.org/licenses/by/4.0/. 
the electric field, the magnetic field and the charge density, which is the basic equation of the electromagnetism in physics. The properties of the singular integral operator and boundary value problems related to the Maxwell equations have been studied by many scholars, for example, Mcintosh A and Mitrea M [17] discussed the problems related to the Maxwell equations in Lipschitz domains. Schneider B and Shapiro M $[18,19]$ studied the Cauchy-type integral of time-harmonic electromagnetic fields in the case of a piecewise Liapunov surface of integration. Kravchenko VV and Shapiro MV [20-22] discussed the Cauchy-type integral associated with Maxwell's equations, and obtained some important integral formulas. Moreover, Kravchenko VV considered quaternionic reformulations of Maxwell's equations and discussed the Dirichlet boundary value problem. Russell DL [23] studied the Dirichlet-Neumann boundary problem associated with the control theory of Maxwell's equations. Yang PW et al. [24] investigated an initial-boundary value problem for Maxwell equations and obtained the general solutions. Colton D and Kress $R$ [25] discussed the boundary value problem for the time-harmonic Maxwell equations and the vector Helmholtz equation. Abreu-Blaya R et al. [26] presented a new definition of Cauchy integral associated with Maxwell equations on 3-dimensional domains with fractal boundaries.

Time-harmonic Maxwell equations in physics are the fundamental equations of electromagnetism and can be rewritten as Helmholtz equations by using the quaternion analysis. Let $\vec{E}, \vec{H}: \Omega \rightarrow \mathbb{C}^{3}$ be a pair of complex-valued vector fields,

$$
\left\{\begin{array}{l}
\operatorname{rot} \vec{H}=\sigma \vec{E}, \quad \operatorname{rot} \vec{E}=i \omega \mu \vec{H} \\
\operatorname{div} \vec{H}=0, \quad \operatorname{div} \vec{E}=0 .
\end{array}\right.
$$

The system is called the time-harmonic Maxwell equations. $(\vec{E}, \vec{H})$ is called a timeharmonic electromagnetic field. It is easy to prove that they satisfy the homogeneous Helmholtz equation

$$
\left\{\begin{array}{l}
\Delta \vec{E}+\lambda \vec{E}=0 \\
\Delta \vec{H}+\lambda \vec{H}=0
\end{array}\right.
$$

where $\lambda=i \omega \mu \sigma \in \mathbb{C}$. In this paper, we will study the Riemann boundary value problem related to the time-harmonic Maxwell equations in quaternion analysis. For the above purpose, we introduce the $\mathcal{N}$ matrix operator which establishes the relationship between the Helmholtz equation and the time-harmonic Maxwell equations. In [15], we discuss some properties of Teodorescu operator and the Riemann boundary value problem related to the Helmholtz equation. By using the $\mathcal{N}$ matrix operator and the conclusions in [15], we give the integral representation of the solution for the Riemann boundary value problem related to the time-harmonic Maxwell equations.

The structure of this paper is as follows: In Sect. 2, we review some basic knowledge of quaternion analysis and introduce some necessary notions for the understanding of this article. In Sect. 3, we first discuss some properties of the singular integral operator $T_{\mathcal{N}, \alpha}$ related to the $\mathcal{N}$ matrix operator, such as uniform boundedness, Hölder continuity and so on. Secondly, we give the integral representation of the solution for the Riemann boundary value problem related to the $\mathcal{N}$ matrix. In Sect. 4, we first introduce the time-harmonic 
Maxwell equations. Then, using the corresponding Cauchy-type integral relationship between the $\mathcal{N}$ matrix operator and the time-harmonic Maxwell equations, we investigate the Riemann boundary value problem related to the time-harmonic Maxwell equations and obtain the integral representation of the solution.

\section{Preliminaries}

Let $\left\{i_{1}, i_{2}, i_{3}\right\}$ be an orthogonal basis of the Euclidean space $R^{3}$, and $\mathbb{H}(\mathbb{C})$ be the set of complex quaternions with basis $\left\{i_{0}, i_{1}, i_{2}, i_{3}\right\}$. Then an arbitrary quaternion $a$ can be written as $a=\sum_{k=0}^{3} a_{k} i_{k}, a_{k} \in \mathbb{C}$, where $i_{0}$ is the unit, $i_{1}, i_{2}, i_{3}$ are the quaternionic imaginary units with the properties

$$
\begin{aligned}
& i_{0}^{2}=-i_{k}^{2}=i_{0}, \quad i_{0} i_{k}=i_{k} i_{0}=i_{k}, \quad k=1,2,3, \\
& i_{1} i_{2}=-i_{2} i_{1}=i_{3}, \quad i_{2} i_{3}=-i_{3} i_{2}=i_{1}, \quad i_{3} i_{1}=-i_{1} i_{3}=i_{2} .
\end{aligned}
$$

The norm for an element $a \in \mathbb{H}(\mathbb{C})$ is taken to be $|a|=\sqrt{\sum_{k=0}^{3}\left|a_{k}\right|^{2}}$. The conjugate operation in $\mathbb{H}(\mathbb{C})$ is governed by the rules

$$
\bar{i}_{0}=i_{0}, \quad \bar{i}_{k}=-i_{k}, \quad k=1,2,3 .
$$

For any complex quaternions $a, b$, we have

$$
a \cdot b=\left(a_{0}+\vec{a}\right) \cdot\left(b_{0}+\vec{b}\right)=a_{0} b_{0}-\langle\vec{a}, \vec{b}\rangle+a_{0} \vec{b}+b_{0} \vec{a}+[\vec{a}, \vec{b}]
$$

where $\langle\vec{a}, \vec{b}\rangle$, $[\vec{a}, \vec{b}]$ stand for usual scalar product and vector product. In particular, $\vec{a} \cdot \vec{b}=$ $-\langle\vec{a}, \vec{b}\rangle+[\vec{a}, \vec{b}]$.

Suppose $\Omega \subset R^{3}$ is a domain with a Liapunov boundary $\partial \Omega$. Then the function which is defined in $\Omega$ and valued in $\mathbb{H}(\mathbb{C})$ can be expressed as $f=\sum_{k=0}^{3} f_{k}(x) i_{k}$, where $f_{k}(x)$ are complex-valued functions. Set

$$
C^{(m)}(\Omega, \mathbb{H}(\mathbb{C}))=\left\{f \mid f: \Omega \rightarrow \mathbb{H}(\mathbb{C}), f(x)=\sum_{k=0}^{3} f_{k}(x) i_{k}, f_{k}(x) \in C^{m}(\Omega, \mathbb{C})\right\} .
$$

We define the differential operators as follows:

$$
\begin{array}{ll}
{ }^{\psi} D[f]=\sum_{k=1}^{3} \psi_{k} \cdot \frac{\partial f}{\partial x_{k}}, & \psi \bar{D}[f]=\sum_{k=1}^{3} \bar{\psi}_{k} \cdot \frac{\partial f}{\partial x_{k}}, \\
D^{\psi}[f]=\sum_{k=1}^{3} \frac{\partial f}{\partial x_{k}} \cdot \psi_{k}, & \bar{D}^{\psi}[f]=\sum_{k=1}^{3} \frac{\partial f}{\partial x_{k}} \cdot \bar{\psi}_{k},
\end{array}
$$

where $\psi=\left\{\psi_{1}, \psi_{2}, \psi_{3}\right\}=\left\{i_{1}, i_{2}, i_{3}\right\}$. For any $f \in C^{(1)}(\Omega, \mathbb{H}(\mathbb{C})), f=f_{0}+\vec{f}$,

$$
\begin{aligned}
{ }^{\psi} D[f] & =\sum_{k=1}^{3} i_{k} \cdot \frac{\partial f}{\partial x_{k}}=\sum_{k=1}^{3} i_{k} \cdot \frac{\partial\left(f_{0}+\vec{f}\right)}{\partial x_{k}} \\
& =\sum_{k=1}^{3} i_{k} \cdot \frac{\partial f_{0}}{\partial x_{k}}-\sum_{k=1}^{3}\left\langle i_{k}, \frac{\partial \vec{f}}{\partial x_{k}}\right\rangle+\sum_{k=1}^{3}\left[i_{k}, \frac{\partial \vec{f}}{\partial x_{k}}\right]
\end{aligned}
$$




$$
=\operatorname{grad} f_{0}-\operatorname{div} \vec{f}+\operatorname{rot} \vec{f} \text {. }
$$

In particular, ${ }^{\psi} D[\vec{f}]=-\operatorname{div} \vec{f}+\operatorname{rot} \vec{f}$.

Let $\lambda \in \mathbb{C} \backslash\{0\}$, and $\alpha$ be its complex square root, $\alpha^{2}=\lambda$. For the above $\alpha$, let us introduce the operators

$$
\begin{array}{ll}
{ }^{\psi} D_{\alpha}[f]=\alpha f+{ }^{\psi} D[f], & { }_{\alpha} D^{\psi}[f]=\alpha f+D^{\psi}[f], \\
{ }^{\psi} \bar{D}_{\alpha}[f]=\alpha f-{ }^{\psi} D[f], & { }_{\alpha} \bar{D}^{\psi}[f]=\alpha f-D^{\psi}[f] .
\end{array}
$$

These are called the left (right) mutually conjugate $(\psi, \alpha)$-hyperholomorphic CauchyRiemann operators. We have the equalities

$$
\begin{aligned}
{ }^{\psi} D_{\alpha}{ }^{\psi} \bar{D}_{\alpha} & ={ }^{\psi} \bar{D}_{\alpha}{ }^{\psi} D_{\alpha}={ }_{\alpha} D^{\psi}{ }_{\alpha} \bar{D}^{\psi}={ }_{\alpha} \bar{D}_{\alpha}^{\psi} D^{\psi} \\
& =\lambda+\triangle_{R^{3}}=\triangle_{\lambda},
\end{aligned}
$$

where $\Delta_{\lambda}$ is the 3-dimensional Helmholtz operator with a complex parameter $\lambda$.

Let $\alpha \in \mathbb{C} \backslash\{0\}$ and $\operatorname{Im} \alpha \neq 0$, we introduce the notation: for $x \in R^{3} \backslash\{0\}$,

$$
\theta_{\alpha}(x)= \begin{cases}-\frac{1}{4 \pi|x|} e^{i \alpha|x|}, & \operatorname{Im} \alpha>0, \\ -\frac{1}{4 \pi|x|} e^{-i \alpha|x|}, & \operatorname{Im} \alpha<0 .\end{cases}
$$

In both cases it is a fundamental solution of the Helmholtz equation with $\lambda=\alpha^{2}$. Then the fundamental solution to the operator ${ }^{\psi} D_{\alpha}, \mathcal{K}_{\psi, \alpha}$ is given by the formula

$$
\mathcal{K}_{\psi, \alpha}(x)=\psi{ }^{\psi} \bar{D}_{\alpha}\left[\theta_{\alpha}\right](x)= \begin{cases}\theta_{\alpha}(x)\left(\alpha+\frac{x}{|x|^{2}}-i \alpha \frac{x}{|x|}\right), & \operatorname{Im} \alpha>0, \\ \theta_{\alpha}(x)\left(\alpha+\frac{x}{|x|^{2}}+i \alpha \frac{x}{|x|}\right), & \operatorname{Im} \alpha<0\end{cases}
$$

An analogous representation holds for $\overline{\mathcal{K}}_{\psi, \alpha}(x)={ }^{\psi} D_{\alpha}\left[\theta_{\alpha}\right](x)$.

If $f$ is a Hölder function, then its $\alpha$-hyperholomorphic Cauchy-type integral is defined by

$$
K_{\psi, \alpha}[f](x)=\int_{\partial \Omega} \mathcal{K}_{\psi, \alpha}(y-x) d \sigma_{y} f(y)
$$

If $f(x) \in L^{p, \sigma}(B, \mathbb{H}(\mathbb{C}))$ means that $f(x) \in L^{p}(B, \mathbb{H}(\mathbb{C})), f^{(\sigma)}(x)=|x|^{-\sigma} f\left(\frac{\bar{x}}{|x|^{2}}\right) \in L^{p}(B, \mathbb{H}(\mathbb{C}))$, in which $B=\{x|| x \mid<1\}, \sigma$ is a real number, and $\|f\|_{p, \sigma}=\|f\|_{L^{p}(B)}+\left\|f^{(\sigma)}\right\|_{L^{p}(B)}, p \geq 1$.

In [15], we introduce the Teodorescu operator related to the Helmholtz equation as follows:

$$
\begin{aligned}
& \left(T_{\psi, \alpha}[f]\right)(x) \\
& =\int_{B} \mathcal{K}_{\psi, \alpha}(y-x) f(y) d v_{y}+\int_{B} \mathcal{K}_{\psi, \alpha}\left(\frac{\bar{y}}{|y|^{2}}-x\right) f\left(\frac{\bar{y}}{|y|^{2}}\right) \frac{1}{|y|^{6}} d v_{y} \\
& =\left(T_{\psi, \alpha}^{(1)}[f]\right)(x)+\left(T_{\psi, \alpha}^{(2)}[f]\right)(x),
\end{aligned}
$$


where $B=\{x|| x \mid<1\}, \alpha=a+i b, b>0$. Analogous representations hold for $\bar{K}_{\psi, \alpha}[f](x)$, $\left(\bar{T}_{\psi, \alpha}[f]\right)(x)$.

In [15], we studied the properties of the above integral operators and obtained the integral representation of the solution for the Riemann boundary value problem related to the Helmholtz equation. The specific results are as follows.

Lemma 2.1 ([15]) Let $B$ be as stated above. If $\in L^{p}(B, \mathbb{H}(\mathbb{C})), 3<p<+\infty$, then

(1) $\left|\left(T_{\psi, \alpha}^{(1)}[f]\right)(x)\right| \leq M_{1}(p)\|f\|_{L^{p}(B)}, x \in R^{3}$,

(2) $\left|\left(T_{\psi, \alpha}^{(1)}[f]\right)\left(x_{1}\right)-\left(T_{\psi, \alpha}^{(1)}[f]\right)\left(x_{2}\right)\right| \leq M_{2}(p)\|f\|_{L^{p}(B)}\left|x_{1}-x_{2}\right|^{\beta}, x_{1}, x_{2} \in \Omega$,

(3) ${ }^{\psi} D_{\alpha}\left(T_{\psi, \alpha}^{(1)}[f]\right)(x)=f(x), x \in B,{ }^{\psi} D_{\alpha}\left(T_{\psi, \alpha}^{(1)}[f]\right)(x)=0, x \in R^{3} \backslash \bar{B}$, where $0<\beta=\frac{p-3}{p}<1$.

Lemma $2.2([15])$ Let $B$ be as stated above. If $\in L^{p}(B, \mathbb{H}(\mathbb{C})), 3<p<+\infty$, then

(1) $\left|\left(\bar{T}_{\psi, \alpha}^{(1)}[f]\right)(x)\right| \leq M_{3}(p)\|f\|_{L^{p}(B)}, x \in R^{3}$,

(2) $\left|\left(\bar{T}_{\psi, \alpha}^{(1)}[f]\right)\left(x_{1}\right)-\left(\bar{T}_{\psi, \alpha}^{(1)}[f]\right)\left(x_{2}\right)\right| \leq M_{4}(p)\|f\|_{L^{p}(B)}\left|x_{1}-x_{2}\right|^{\beta}, x_{1}, x_{2} \in \Omega$,

(3) ${ }^{\psi} \bar{D}_{\alpha}\left(\bar{T}_{\psi, \alpha}^{(1)}[f]\right)(x)=f(x), x \in B,{ }^{\psi} \bar{D}_{\alpha}\left(T_{\psi, \alpha}^{(1)}[f]\right)(x)=0, x \in R^{3} \backslash \bar{B}$, where $0<\beta=\frac{p-3}{p}<1$.

Remark 2.1 Analogous properties hold for $T_{\psi, \alpha}^{(2)}, T_{\psi, \alpha}, \bar{T}_{\psi, \alpha}^{(2)}, \bar{T}_{\psi, \alpha}$. For more information, we refer the reader to [15].

Lemma 2.3 ([15]) Let $B$ be as stated above. Find a quaternion-valued function $u(x)$ satisfying the system ${ }^{\psi} D_{\alpha}[u]=0\left(x \in R^{3} \backslash \partial B\right)$ and vanishing at infinity with the boundary condition

$$
u^{+}(\tau)=u^{-}(\tau) G+f(\tau), \quad \tau \in \partial B
$$

where $u^{ \pm}(\tau)=\lim _{x \in B^{ \pm}, x \rightarrow \tau} u(x), G$ is a quaternion constant, $G^{-1}$ exists, and $f \in H_{\partial B}^{v}(0<$ $v<1)$. Then the solution can be expressed as

$$
u(x)= \begin{cases}\int_{\partial B} \mathcal{K}_{\psi, \alpha}(y-x) d \sigma_{y} f(y), & x \in B^{+}, \\ \int_{\partial B} \mathcal{K}_{\psi, \alpha}(y-x) d \sigma_{y} f(y) G^{-1}, & x \in B^{-} .\end{cases}
$$

\section{Some properties and applications of the Teodorescu operator $T_{\mathcal{N}, \alpha}$ related to the $\mathcal{N}$ matrix operator}

\subsection{The relevant definitions and symbols}

We will consider the following matrix operator:

$$
\mathcal{N}=\left(\begin{array}{cc}
\sigma & -{ }^{\psi} D \\
{ }^{\psi} D & -i \omega \mu
\end{array}\right)
$$

where $\alpha^{2}=i \omega \mu \sigma$. We shall consider it on the set $C^{(1)}\left(\Omega\right.$, $\left.\operatorname{Mat}_{2 \times 2}(\mathbb{H}(\mathbb{C}))\right), \operatorname{Mat}_{2 \times 2}(\mathbb{H}(\mathbb{C}))$ being the set of $2 \times 2$ matrices with entries from $\mathbb{H}(\mathbb{C})$.

Let

$$
A_{1}=\left(\begin{array}{cc}
\alpha & -\sigma \\
-\alpha & -\sigma
\end{array}\right), \quad B_{1}=\frac{1}{2}\left(\begin{array}{cc}
\sigma^{-1} & -\sigma^{-1} \\
\alpha^{-1} & \alpha^{-1}
\end{array}\right),
$$


then

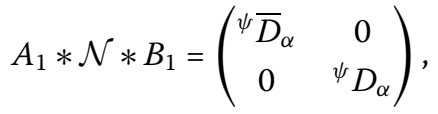

where " $*$ " stands for matrix multiplication.

Analogously, let

$$
A_{2}=\left(\begin{array}{cc}
-\alpha & -\sigma \\
\alpha & -\sigma
\end{array}\right), \quad B_{2}=\frac{1}{2}\left(\begin{array}{cc}
-\sigma^{-1} & \sigma^{-1} \\
\alpha^{-1} & \alpha^{-1}
\end{array}\right),
$$

then

$$
A_{2} * \mathcal{N} * B_{2}=\left(\begin{array}{cc}
{ }^{\psi} D_{\alpha} & 0 \\
0 & \psi \bar{D}_{\alpha}
\end{array}\right),
$$

where $A_{1}, B_{1}, A_{2}, B_{2}$ are invertible.

Thus there exist invertible matrices of $A_{1}, B_{1}, A_{2}, B_{2}$ such that

$$
\mathcal{N}=A_{1}^{-1} *\left(\begin{array}{cc}
\psi \bar{D}_{\alpha} & 0 \\
0 & { }^{\psi} D_{\alpha}
\end{array}\right) * B_{1}^{-1}
$$

and

$$
\mathcal{N}=A_{2}^{-1} *\left(\begin{array}{cc}
{ }^{\psi} D_{\alpha} & 0 \\
0 & \psi \bar{D}_{\alpha}
\end{array}\right) * B_{2}^{-1} .
$$

For $\alpha \in \mathbb{C} \backslash\{0\}$, let

$$
\mathcal{K}_{\mathcal{N}, \alpha}=B_{1} * A_{2} * \mathcal{N} * B_{2} * A_{1} *\left(\begin{array}{cc}
\theta_{\alpha} & 0 \\
0 & \theta_{\alpha}
\end{array}\right)
$$

be the quaternionic Cauchy-Maxwell kernel, which is the fundamental solution of $\mathcal{N}$ operator. The reasons are as follows. By the definition of $\mathcal{N}$ operator, we have

$$
\begin{aligned}
\mathcal{K}_{\mathcal{N}, \alpha} & =B_{1} * A_{2} * A_{2}{ }^{-1} *\left(\begin{array}{cc}
{ }^{\psi} D_{\alpha} & 0 \\
0 & \psi \bar{D}_{\alpha}
\end{array}\right) * B_{2}{ }^{-1} * B_{2} * A_{1} *\left(\begin{array}{cc}
\theta_{\alpha} & 0 \\
0 & \theta_{\alpha}
\end{array}\right) \\
& =B_{1} *\left(\begin{array}{cc}
{ }^{\psi} D_{\alpha} & 0 \\
0 & \psi \\
{ }^{\prime} & \bar{D}_{\alpha}
\end{array}\right) * A_{1} *\left(\begin{array}{cc}
\theta_{\alpha} & 0 \\
0 & \theta_{\alpha}
\end{array}\right) .
\end{aligned}
$$

By $\Delta_{\lambda}\left[\theta_{\alpha}\right]={ }^{\psi} D_{\alpha} \psi \bar{D}_{\alpha}\left[\theta_{\alpha}\right]={ }^{\psi} \bar{D}_{\alpha}{ }^{\psi} D_{\alpha}\left[\theta_{\alpha}\right]=0$, we have

$$
\begin{aligned}
& \mathcal{N}\left[\mathcal{K}_{\mathcal{N}, \alpha}\right] \\
& \quad=A_{1}{ }^{-1} *\left(\begin{array}{cc}
{ }^{\psi} \bar{D}_{\alpha} & 0 \\
0 & { }^{\psi} D_{\alpha}
\end{array}\right) * B_{1}{ }^{-1} * B_{1} *\left(\begin{array}{cc}
{ }^{\psi} D_{\alpha} & 0 \\
0 & \psi \bar{D}_{\alpha}
\end{array}\right) * A_{1} *\left(\begin{array}{cc}
\theta_{\alpha} & 0 \\
0 & \theta_{\alpha}
\end{array}\right) \\
& \quad=A_{1}{ }^{-1} *\left(\begin{array}{cc}
{ }^{\psi} \bar{D}_{\alpha} & 0 \\
0 & { }^{\psi} D_{\alpha}
\end{array}\right) *\left(\begin{array}{cc}
{ }^{\psi} D_{\alpha} & 0 \\
0 & \psi \bar{D}_{\alpha}
\end{array}\right) * A_{1} *\left(\begin{array}{cc}
\theta_{\alpha} & 0 \\
0 & \theta_{\alpha}
\end{array}\right)
\end{aligned}
$$




$$
\begin{aligned}
& =A_{1}{ }^{-1} *\left(\begin{array}{cc}
{ }^{\psi} \bar{D}_{\alpha} & 0 \\
0 & { }^{\psi} D_{\alpha}
\end{array}\right) *\left(\begin{array}{cc}
{ }^{\psi} D_{\alpha} & 0 \\
0 & \psi \\
\bar{D}_{\alpha}
\end{array}\right) *\left(\begin{array}{cc}
\alpha & -\sigma \\
-\alpha & -\sigma
\end{array}\right) *\left(\begin{array}{cc}
\theta_{\alpha} & 0 \\
0 & \theta_{\alpha}
\end{array}\right) \\
& =A_{1}{ }^{-1} *\left(\begin{array}{cl}
\alpha^{\psi} \bar{D}_{\alpha}{ }^{\psi} D_{\alpha}\left[\theta_{\alpha}\right] & -\sigma^{\psi} \bar{D}_{\alpha}{ }^{\psi} D_{\alpha}\left[\theta_{\alpha}\right] \\
-\alpha^{\psi} D_{\alpha}{ }^{\psi} \bar{D}_{\alpha}\left[\theta_{\alpha}\right] & -\sigma^{\psi} D_{\alpha}{ }^{\psi} \bar{D}_{\alpha}\left[\theta_{\alpha}\right]
\end{array}\right)=0 .
\end{aligned}
$$

Let

$$
K_{\mathcal{N}, \alpha}[f](x)=\int_{\partial \Omega} \mathcal{K}_{\mathcal{N}, \alpha}(y-x) * \tilde{d} \sigma_{y} * f(y), \quad x \in \Omega^{ \pm}
$$

be an analog of the Cauchy-type integral in the theory of the integral representations with the quaternionic Cauchy-Maxwell kernel, where $f: \partial \Omega \rightarrow \operatorname{Mat}_{2 \times 2}(\mathbb{H}(\mathbb{C}))$ and

$$
\tilde{d} \sigma_{y}=\left(\begin{array}{cc}
0 & -d \sigma_{y} \\
d \sigma_{y} & 0
\end{array}\right)
$$

We shall call also $K_{\mathcal{N}, \alpha}[f](x)$ the quaternionic Cauchy-Maxwell-type integral.

The norm of an matrix $f=\left(\begin{array}{l}f_{11} f_{12} \\ f_{21} f_{22}\end{array}\right) \in \operatorname{Mat}_{2 \times 2}(\mathbb{H}(\mathbb{C}))$ is taken to be $\|f\|_{\infty}=\max _{1 \leq i, j \leq 2}\left|f_{i j}\right|$. From equality (3.1), we know there exists a direct connection between $\mathcal{K}_{\mathcal{N}, \alpha}$ and the corresponding hyperholomorphic Cauchy kernels $\mathcal{K}_{\psi, \alpha}, \overline{\mathcal{K}}_{\psi, \alpha}$.

$$
\begin{aligned}
\mathcal{K}_{\mathcal{N}, \alpha}(x) & =\frac{1}{2}\left(\begin{array}{cc}
\sigma^{-1} & -\sigma^{-1} \\
\alpha^{-1} & \alpha^{-1}
\end{array}\right) *\left(\begin{array}{cc}
{ }^{\psi} D_{\alpha} & 0 \\
0 & \psi \\
\bar{D}_{\alpha}
\end{array}\right) *\left(\begin{array}{cc}
\alpha & -\sigma \\
-\alpha & -\sigma
\end{array}\right) *\left(\begin{array}{cc}
\theta_{\alpha} & 0 \\
0 & \theta_{\alpha}
\end{array}\right) \\
& =\frac{1}{2}\left(\begin{array}{cc}
\sigma^{-1} \alpha\left(\overline{\mathcal{K}}_{\psi, \alpha}(x)+\mathcal{K}_{\psi, \alpha}(x)\right) & -\left(\overline{\mathcal{K}}_{\psi, \alpha}(x)-\mathcal{K}_{\psi, \alpha}(x)\right) \\
\overline{\mathcal{K}}_{\psi, \alpha}(x)-\mathcal{K}_{\psi, \alpha}(x) & \left.-\alpha^{-1} \sigma \overline{\mathcal{K}}_{\psi, \alpha}(x)+\mathcal{K}_{\psi, \alpha}(x)\right)
\end{array}\right) \\
& =\frac{1}{2}\left(\begin{array}{cc}
\sigma^{-1} & \sigma^{-1} \\
\alpha^{-1} & -\alpha^{-1}
\end{array}\right) *\left(\begin{array}{cc}
\alpha \overline{\mathcal{K}}_{\psi, \alpha}(x) & -\sigma \overline{\mathcal{K}}_{\psi, \alpha}(x) \\
\alpha \mathcal{K}_{\psi, \alpha}(x) & \sigma \mathcal{K}_{\psi, \alpha}(x)
\end{array}\right),
\end{aligned}
$$

where $\mathcal{K}_{\psi, \alpha}(x), \overline{\mathcal{K}}_{\psi, \alpha}(x)$ are, respectively, the Cauchy kernel for ${ }^{\psi} D_{\alpha},{ }^{\psi} \bar{D}_{\alpha}$.

\subsection{Some properties of the Teodorescu operator $T_{\mathcal{N}, \alpha}$ related to the $\mathcal{N}$ matrix operator}

In this section, we will discuss some properties of the following singular integral operators:

$$
\begin{aligned}
& \left(T_{\mathcal{N}, \alpha}[f]\right)(x) \\
& =\int_{B} \mathcal{K}_{\mathcal{N}, \alpha}(y-x) * f(y) d v_{y}+\int_{B} \mathcal{K}_{\mathcal{N}, \alpha}\left(\frac{\bar{y}}{|y|^{2}}-x\right) * f\left(\frac{\bar{y}}{|y|^{2}}\right) * \frac{1}{|y|^{6}} d v_{y} \\
& =\left(T_{\mathcal{N}, \alpha}^{(1)}[f]\right)(x)+\left(T_{\mathcal{N}, \alpha}^{(2)}[f]\right)(x),
\end{aligned}
$$

where $B=\{x|| x \mid<1\}, \alpha=a+i b, b>0$.

Theorem 3.1 Let $\Omega, B, \alpha$ be as stated above. If $f=\left(\begin{array}{l}f_{11} f_{12} \\ f_{21} f_{22}\end{array}\right)$ with entries belonging to $L^{p}(B, \mathbb{H}(\mathbb{C})), 3<p<+\infty$, then

(1) $\left\|\left(T_{\mathcal{N}, \alpha}^{(1)}[f]\right)(x)\right\|_{\infty} \leq Q_{1}(p) \cdot \max _{1 \leq i, j \leq 2}\left\|f_{i j}\right\|_{L^{p}(B)}, x \in R^{3}$,

(2) $\left\|\left(T_{\mathcal{N}, \alpha}^{(1)}[f]\right)\left(x_{1}\right)-\left(T_{\mathcal{N}, \alpha}^{(1)}[f]\right)\left(x_{2}\right)\right\|_{\infty} \leq Q_{2}(p) \cdot \max _{1 \leq i, j \leq 2}\left\|f_{i j}\right\|_{L^{p}(B)} \cdot\left|x_{1}-x_{2}\right|^{\beta}, x_{1}, x_{2} \in \Omega$, 
(3) $\mathcal{N}\left(T_{\mathcal{N}, \alpha}^{(1)}[f]\right)(x)=f(x), x \in B, \mathcal{N}\left(T_{\mathcal{N}, \alpha}^{(1)}[f]\right)(x)=0, x \in R^{3} \backslash \bar{B}$, where $0<\beta=\frac{p-3}{p}<1$.

Proof (1) From (3.2), we can obtain

$$
\begin{aligned}
\left(T_{\mathcal{N}, \alpha}^{(1)}[f]\right)(x) & \\
\quad & \int_{B} \mathcal{K}_{\mathcal{N}, \alpha}(y-x) * f(y) d v_{y} \\
& =\frac{1}{2} \int_{B}\left(\begin{array}{cc}
\sigma^{-1} & \sigma^{-1} \\
\alpha^{-1} & -\alpha^{-1}
\end{array}\right) *\left(\begin{array}{cc}
\alpha \overline{\mathcal{K}}_{\psi, \alpha} & -\sigma \overline{\mathcal{K}}_{\psi, \alpha} \\
\alpha \mathcal{K}_{\psi, \alpha} & \sigma \mathcal{K}_{\psi, \alpha}
\end{array}\right) *\left(\begin{array}{ll}
f_{11} & f_{12} \\
f_{21} & f_{22}
\end{array}\right) d v_{y} \\
& =\frac{1}{2} \int_{B}\left(\begin{array}{cc}
\sigma^{-1} & \sigma^{-1} \\
\alpha^{-1} & -\alpha^{-1}
\end{array}\right) *\left(\begin{array}{ll}
\alpha \overline{\mathcal{K}}_{\psi, \alpha} f_{11}-\sigma \overline{\mathcal{K}}_{\psi, \alpha} f_{21} & \alpha \overline{\mathcal{K}}_{\psi, \alpha} f_{12}-\sigma \overline{\mathcal{K}}_{\psi, \alpha} f_{22} \\
\alpha \mathcal{K}_{\psi, \alpha} f_{11}+\sigma \mathcal{K}_{\psi, \alpha} f_{21} & \alpha \mathcal{K}_{\psi, \alpha} f_{12}+\sigma \mathcal{K}_{\psi, \alpha} f_{22}
\end{array}\right) d v_{y} \\
& =\frac{1}{2}\left(\begin{array}{ll}
g_{11} & g_{12} \\
g_{21} & g_{22}
\end{array}\right)
\end{aligned}
$$

where

$$
\begin{aligned}
& g_{11}=\alpha \sigma^{-1} \int_{B}\left[\overline{\mathcal{K}}_{\psi, \alpha}+\mathcal{K}_{\psi, \alpha}\right] f_{11} d v_{y}+\int_{B}\left[\mathcal{K}_{\psi, \alpha}-\overline{\mathcal{K}}_{\psi, \alpha}\right] f_{21} d v_{y}, \\
& g_{12}=\alpha \sigma^{-1} \int_{B}\left[\overline{\mathcal{K}}_{\psi, \alpha}+\mathcal{K}_{\psi, \alpha}\right] f_{12} d v_{y}+\int_{B}\left[\mathcal{K}_{\psi, \alpha}-\overline{\mathcal{K}}_{\psi, \alpha}\right] f_{22} d v_{y}, \\
& g_{21}=\int_{B}\left[\overline{\mathcal{K}}_{\psi, \alpha}-\mathcal{K}_{\psi, \alpha}\right] f_{11} d v_{y}-\alpha^{-1} \sigma \int_{B}\left[\overline{\mathcal{K}}_{\psi, \alpha}+\mathcal{K}_{\psi, \alpha}\right] f_{21} d v_{y}, \\
& g_{22}=\int_{B}\left[\overline{\mathcal{K}}_{\psi, \alpha}-\mathcal{K}_{\psi, \alpha}\right] f_{12} d v_{y}-\alpha^{-1} \sigma \int_{B}\left[\overline{\mathcal{K}}_{\psi, \alpha}+\mathcal{K}_{\psi, \alpha}\right] f_{22} d v_{y} .
\end{aligned}
$$

By Lemma 2.1 and Lemma 2.2, we have

$$
\begin{aligned}
& \left|g_{11}\right| \leq Q_{1}^{(1)}(p)\left\|f_{11}\right\|_{L^{p}(B)}+Q_{1}^{(2)}(p)\left\|f_{21}\right\|_{L^{p}(B)}, \\
& \left|g_{12}\right| \leq Q_{1}^{(3)}(p)\left\|f_{12}\right\|_{L^{p}(B)}+Q_{1}^{(4)}(p)\left\|f_{22}\right\|_{L^{p}(B)}, \\
& \left|g_{21}\right| \leq Q_{1}^{(5)}(p)\left\|f_{11}\right\|_{L^{p}(B)}+Q_{1}^{(6)}(p)\left\|f_{21}\right\|_{L^{p}(B)}, \\
& \left|g_{22}\right| \leq Q_{1}^{(7)}(p)\left\|f_{12}\right\|_{L^{p}(B)}+Q_{1}^{(8)}(p)\left\|f_{22}\right\|_{L^{p}(B)} .
\end{aligned}
$$

Therefore

$$
\left\|\left(T_{\mathcal{N}, \alpha}^{(1)}[f]\right)(x)\right\|_{\infty}=\frac{1}{2} \max _{1 \leq i, j \leq 2}\left|g_{i j}\right| \leq Q_{1}(p) \cdot \max _{1 \leq i, j \leq 2}\left\|f_{i j}\right\|_{L^{p}(B)},
$$

where $Q_{1}(p)=\max _{1 \leq i \leq 8}\left\{Q_{1}^{(i)}(p)\right\}$.

(2) From (3.3), we can obtain

$$
\left(T_{\mathcal{N}, \alpha}^{(1)}[f]\right)\left(x_{1}\right)-\left(T_{\mathcal{N}, \alpha}^{(1)}[f]\right)\left(x_{2}\right)=\frac{1}{2}\left(\begin{array}{ll}
g_{11}\left(x_{1}\right)-g_{11}\left(x_{2}\right) & g_{12}\left(x_{1}\right)-g_{12}\left(x_{2}\right) \\
g_{21}\left(x_{1}\right)-g_{21}\left(x_{2}\right) & g_{22}\left(x_{1}\right)-g_{22}\left(x_{2}\right)
\end{array}\right) .
$$


For each $x_{1}, x_{2} \in \Omega$, by Lemma 2.1 and Lemma 2.2, we have

$$
\begin{aligned}
& \left|g_{11}\left(x_{1}\right)-g_{11}\left(x_{2}\right)\right| \leq Q_{2}^{(1)}(p)\left\|f_{11}\right\|_{L^{p}(B)}\left|x_{1}-x_{2}\right|^{\beta}+Q_{2}^{(2)}(p)\left\|f_{21}\right\|_{L^{p}(B)}\left|x_{1}-x_{2}\right|^{\beta}, \\
& \left|g_{12}\left(x_{1}\right)-g_{12}\left(x_{2}\right)\right| \leq Q_{2}^{(3)}(p)\left\|f_{12}\right\|_{L^{p}(B)}\left|x_{1}-x_{2}\right|^{\beta}+Q_{2}^{(4)}(p)\left\|f_{22}\right\|_{L^{p}(B)}\left|x_{1}-x_{2}\right|^{\beta}, \\
& \left|g_{21}\left(x_{1}\right)-g_{21}\left(x_{2}\right)\right| \leq Q_{2}^{(5)}(p)\left\|f_{11}\right\|_{L^{p}(B)}\left|x_{1}-x_{2}\right|^{\beta}+Q_{2}^{(6)}(p)\left\|f_{21}\right\|_{L^{p}(B)}\left|x_{1}-x_{2}\right|^{\beta}, \\
& \left|g_{22}\left(x_{1}\right)-g_{22}\left(x_{2}\right)\right| \leq Q_{2}^{(7)}(p)\left\|f_{12}\right\|_{L^{p}(B)}\left|x_{1}-x_{2}\right|^{\beta}+Q_{2}^{(8)}(p)\left\|f_{22}\right\|_{L^{p}(B)}\left|x_{1}-x_{2}\right|^{\beta} .
\end{aligned}
$$

Therefore

$$
\begin{aligned}
& \left\|\left(T_{\mathcal{N}, \alpha}^{(1)}[f]\right)\left(x_{1}\right)-\left(T_{\mathcal{N}, \alpha}^{(1)}[f]\right)\left(x_{2}\right)\right\|_{\infty} \\
& \quad=\frac{1}{2} \max _{1 \leq i, j \leq 2}\left|g_{i j}\left(x_{1}\right)-g_{i j}\left(x_{2}\right)\right| \\
& \quad \leq Q_{2}(p) \cdot \max _{1 \leq i, j \leq 2}\left\|f_{i j}\right\|_{L^{p}(B)}\left|x_{1}-x_{2}\right|^{\beta},
\end{aligned}
$$

where $Q_{2}(p)=\max _{1 \leq i \leq 8}\left\{Q_{2}^{(i)}(p)\right\}$.

(3) From (3.3), we can obtain

$$
\begin{aligned}
\mathcal{N}\left(T_{\mathcal{N}, \alpha}^{(1)}[f]\right)(x) & =\left(\begin{array}{cc}
\sigma & -{ }^{\psi} D \\
{ }^{\psi} D & -i \omega \mu
\end{array}\right) * \frac{1}{2}\left(\begin{array}{ll}
g_{11} & g_{12} \\
g_{21} & g_{22}
\end{array}\right) \\
& =\frac{1}{2}\left(\begin{array}{cc}
\sigma g_{11}-{ }^{\psi} D\left[g_{21}\right] & \sigma g_{12}-{ }^{\psi} D\left[g_{22}\right] \\
{ }^{\psi} D\left[g_{11}\right]-i \omega \mu g_{21} & { }^{\psi} D\left[g_{12}\right]-i \omega \mu g_{22}
\end{array}\right) .
\end{aligned}
$$

Thus

$$
\begin{aligned}
& \sigma g_{11}-{ }^{\psi} D\left[g_{21}\right] \\
& =\alpha \int_{B}\left[\overline{\mathcal{K}}_{\psi, \alpha}+\mathcal{K}_{\psi, \alpha}\right] f_{11} d v_{y}+\sigma \int_{B}\left[\mathcal{K}_{\psi, \alpha}-\overline{\mathcal{K}}_{\psi, \alpha}\right] f_{21} d v_{y} \\
& \quad-{ }^{\psi} D\left\{\int_{B}\left[\overline{\mathcal{K}}_{\psi, \alpha}-\mathcal{K}_{\psi, \alpha}\right] f_{11} d v_{y}\right\}+\alpha^{-1} \sigma^{\psi} D\left\{\int_{B}\left[\overline{\mathcal{K}}_{\psi, \alpha}+\mathcal{K}_{\psi, \alpha}\right] f_{21} d v_{y}\right\} \\
& ={ }^{\psi} D_{\alpha}\left\{\int_{B} \mathcal{K}_{\psi, \alpha} f_{11} d v_{y}\right\}+{ }^{\psi} \bar{D}_{\alpha}\left\{\int_{B} \overline{\mathcal{K}}_{\psi, \alpha} f_{11} d v_{y}\right\} \\
& \quad+\sigma \alpha^{-1 \psi} D_{\alpha}\left\{\int_{B} \mathcal{K}_{\psi, \alpha} f_{21} d v_{y}\right\}-\sigma \alpha^{-1 \psi} \bar{D}_{\alpha}\left\{\int_{B} \overline{\mathcal{K}}_{\psi, \alpha} f_{21} d v_{y}\right\},
\end{aligned}
$$

by Lemma 2.1 and Lemma 2.2, we have

$$
\sigma g_{11}-{ }^{\psi} D\left[g_{21}\right]=2 f_{11}, \quad x \in B, \quad \sigma g_{11}-{ }^{\psi} D\left[g_{21}\right]=0, \quad x \in R^{3} \backslash \bar{B} .
$$

Similarly, we can obtain

$$
\begin{aligned}
& \sigma g_{12}-{ }^{\psi} D\left[g_{22}\right]=2 f_{12}, \quad x \in B, \quad \sigma g_{12}-{ }^{\psi} D\left[g_{22}\right]=0, \quad x \in R^{3} \backslash \bar{B}, \\
& { }^{\psi} D\left[g_{11}\right]-i \omega \mu g_{21}=2 f_{21}, \quad x \in B, \quad{ }^{\psi} D\left[g_{11}\right]-i \omega \mu g_{21}=0, \quad x \in R^{3} \backslash \bar{B},
\end{aligned}
$$




$$
{ }^{\psi} D\left[g_{12}\right]-i \omega \mu g_{22}=2 f_{22}, \quad x \in B, \quad{ }^{\psi} D\left[g_{12}\right]-i \omega \mu g_{22}=0, \quad x \in R^{3} \backslash \bar{B} .
$$

Therefore $\mathcal{N}\left(T_{\mathcal{N}, \alpha}^{(1)}[f]\right)(x)=f(x), x \in B, \mathcal{N}\left(T_{\mathcal{N}, \alpha}^{(1)}[f]\right)(x)=0, x \in R^{3} \backslash \bar{B}$.

Theorem 3.2 Let $\Omega, B, \alpha$ be as stated above. If $f=\left(\begin{array}{l}f_{11} f_{12} \\ f_{21} f_{22}\end{array}\right)$ with entries belonging to $L^{p, 3}(B, \mathbb{H}(\mathbb{C})), 3<p<+\infty$, then

(1) $\left\|\left(T_{\mathcal{N}, \alpha}^{(2)}[f]\right)(x)\right\|_{\infty} \leq Q_{3}(p) \cdot \max _{1 \leq i, j \leq 2}\left\|f_{i j}^{(3)}\right\|_{L^{p}(B)}, x \in R^{3}$,

(2) $\left\|\left(T_{\mathcal{N}, \alpha}^{(2)}[f]\right)\left(x_{1}\right)-\left(T_{\mathcal{N}, \alpha}^{(2)}[f]\right)\left(x_{2}\right)\right\|_{\infty} \leq Q_{4}(p) \cdot \max _{1 \leq i, j \leq 2}\left\|f_{i j}^{(3)}\right\|_{L^{p}(B)} \cdot\left|x_{1}-x_{2}\right|^{\beta}$, $x_{1}, x_{2} \in \Omega$,

(3) $\mathcal{N}\left(T_{\mathcal{N}, \alpha}^{(2)}[f]\right)(x)=0, x \in B, \mathcal{N}\left(T_{\mathcal{N}, \alpha}^{(2)}[f]\right)(x)=f(x), x \in R^{3} \backslash \bar{B}$, where $0<\beta=\frac{p-3}{p}<1$.

Proof This case is similar to Theorem 3.1.

Thus, from Theorem 3.1 and Theorem 3.2, we obtain the following results.

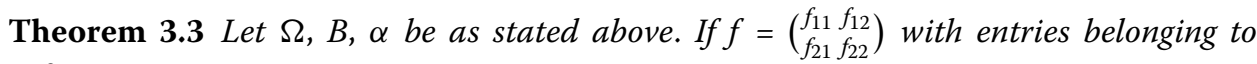
$L^{p, 3}(B, \mathbb{H}(\mathbb{C})), 3<p<+\infty$, then

(1) $\left\|\left(T_{\mathcal{N}, \alpha}[f]\right)(x)\right\|_{\infty} \leq Q_{5}(p) \cdot \max _{1 \leq i, j \leq 2}\left\|f_{i j}\right\|_{p, 3}, x \in R^{3}$,

(2) $\left\|\left(T_{\mathcal{N}, \alpha}[f]\right)\left(x_{1}\right)-\left(T_{\mathcal{N}, \alpha}[f]\right)\left(x_{2}\right)\right\|_{\infty} \leq Q_{6}(p) \cdot \max _{1 \leq i, j \leq 2}\left\|f_{i j}\right\|_{p, 3} \cdot\left|x_{1}-x_{2}\right|^{\beta}, x_{1}, x_{2} \in \Omega$,

(3) $\mathcal{N}\left(T_{\mathcal{N}, \alpha}[f]\right)(x)=f(x), x \in R^{3} \backslash \partial B$,

where $0<\beta=\frac{p-3}{p}<1$.

\subsection{The Riemann boundary value problem related to the $\mathcal{N}$ matrix operator}

Theorem 3.4 Let $B$ be as stated above. Find $u=\left(\begin{array}{ll}u_{11} & u_{12} \\ u_{21} & u_{22}\end{array}\right) \in \operatorname{Mat}_{2 \times 2}(\mathbb{H}(\mathbb{C}))$ satisfying the following system:

$$
\left\{\begin{array}{l}
\mathcal{N}[u]=0, \quad x \in R^{3} \backslash \partial B, \\
u^{+}(\tau)=u^{-}(\tau) * G+f(\tau), \quad \tau \in \partial B, \\
u(x) \rightarrow 0, \quad \text { as } x \rightarrow \infty,
\end{array}\right.
$$

where $u^{ \pm}(\tau)=\lim _{x \in B^{ \pm}, x \rightarrow \tau} u(x), G=\left(\begin{array}{cc}G_{11} & 0 \\ 0 & G_{22}\end{array}\right)$ is a quaternion constant matrix and its inverse exists, $f=\left(\begin{array}{ll}f_{11} & f_{12} \\ f_{21} & f_{22}\end{array}\right)$ with entries belonging to $H_{\partial B}^{v}(0<v<1)$. Then the solution can be expressed as

$$
u(x)= \begin{cases}\int_{\partial B} \mathcal{K}_{\mathcal{N}, \alpha}(y-x) * \tilde{d} \sigma_{y} * f(y), & x \in B^{+} \\ \int_{\partial B} \mathcal{K}_{\mathcal{N}, \alpha}(y-x) * \tilde{d} \sigma_{y} * f(y) * G^{-1}, & x \in B^{-}\end{cases}
$$

Proof Let $B_{2}$ be as above and its inverse $B_{2}^{-1}=\left(\begin{array}{cc}-\sigma & \alpha \\ \sigma & \alpha\end{array}\right)$. Then, for $g=B_{2}^{-1} u=\left(\begin{array}{ll}g_{11} & g_{12} \\ g_{21} & g_{22}\end{array}\right)$, since $\mathcal{N}[u]=0$, we can obtain

$$
{ }^{\psi} D_{\alpha}\left[g_{11}\right]={ }^{\psi} D_{\alpha}\left[g_{12}\right]=0, \quad \psi \bar{D}_{\alpha}\left[g_{21}\right]=\psi \bar{D}_{\alpha}\left[g_{22}\right]=0
$$

By (3.4), we have $g^{+}=g^{-} * G+B_{2}^{-1} * f$, i.e.

$$
g_{11}{ }^{+}=g_{11}{ }^{-} G_{11}-\sigma f_{11}+\alpha f_{21}, \quad g_{12}{ }^{+}=g_{12}{ }^{-} G_{22}-\sigma f_{12}+\alpha f_{22},
$$




$$
g_{21}{ }^{+}=g_{21}{ }^{-} G_{11}+\sigma f_{11}+\alpha f_{21}, \quad g_{22}{ }^{+}=g_{22}{ }^{-} G_{22}+\sigma f_{12}+\alpha f_{22} .
$$

By Lemma 2.3, when $x \in B^{+}$,

$$
g=\left(\begin{array}{cc}
-\sigma K_{\psi, \alpha}\left[f_{11}\right]+\alpha K_{\psi, \alpha}\left[f_{21}\right] & -\sigma K_{\psi, \alpha}\left[f_{12}\right]+\alpha K_{\psi, \alpha}\left[f_{22}\right] \\
\sigma \bar{K}_{\psi, \alpha}\left[f_{11}\right]+\alpha \bar{K}_{\psi, \alpha}\left[f_{21}\right] & \sigma \bar{K}_{\psi, \alpha}\left[f_{12}\right]+\alpha \bar{K}_{\psi, \alpha}\left[f_{22}\right]
\end{array}\right) .
$$

Then

$$
\begin{aligned}
u & =B_{2} * g \\
& =\frac{1}{2}\left(\begin{array}{cc}
-\sigma^{-1} & \sigma^{-1} \\
\alpha^{-1} & \alpha^{-1}
\end{array}\right) *\left(\begin{array}{cc}
-\sigma K_{\psi, \alpha}\left[f_{11}\right]+\alpha K_{\psi, \alpha}\left[f_{21}\right] & -\sigma K_{\psi, \alpha}\left[f_{12}\right]+\alpha K_{\psi, \alpha}\left[f_{22}\right] \\
\sigma \bar{K}_{\psi, \alpha}\left[f_{11}\right]+\alpha \bar{K}_{\psi, \alpha}\left[f_{21}\right] & \sigma \bar{K}_{\psi, \alpha}\left[f_{12}\right]+\alpha \bar{K}_{\psi, \alpha}\left[f_{22}\right]
\end{array}\right) \\
& =\int_{\partial B} \mathcal{K}_{\mathcal{N}, \alpha}(y-x) * \tilde{d} \sigma_{y} * f(y) .
\end{aligned}
$$

By Lemma 2.3, when $x \in B^{-}$,

$$
\begin{aligned}
g & =\left(\begin{array}{cc}
-\sigma K_{\psi, \alpha}\left[f_{11}\right] G_{11}^{-1}+\alpha K_{\psi, \alpha}\left[f_{21}\right] G_{11}^{-1} & -\sigma K_{\psi, \alpha}\left[f_{12}\right] G_{22}^{-1}+\alpha K_{\psi, \alpha}\left[f_{22}\right] G_{22}^{-1} \\
\sigma \bar{K}_{\psi, \alpha}\left[f_{11}\right] G_{11}^{-1}+\alpha \bar{K}_{\psi, \alpha}\left[f_{21}\right] G_{11}^{-1} & \sigma \bar{K}_{\psi, \alpha}\left[f_{12}\right] G_{22}^{-1}+\alpha \bar{K}_{\psi, \alpha}\left[f_{22}\right] G_{22}^{-1}
\end{array}\right) \\
& =\left(\begin{array}{cc}
-\sigma K_{\psi, \alpha}\left[f_{11}\right]+\alpha K_{\psi, \alpha}\left[f_{21}\right] & -\sigma K_{\psi, \alpha}\left[f_{12}\right]+\alpha K_{\psi, \alpha}\left[f_{22}\right] \\
\sigma \bar{K}_{\psi, \alpha}\left[f_{11}\right]+\alpha \bar{K}_{\psi, \alpha}\left[f_{21}\right] & \sigma \bar{K}_{\psi, \alpha}\left[f_{12}\right]+\alpha \bar{K}_{\psi, \alpha}\left[f_{22}\right]
\end{array}\right) *\left(\begin{array}{cc}
G_{11}^{-1} & 0 \\
0 & G_{22}^{-1}
\end{array}\right) .
\end{aligned}
$$

Then

$$
u=B_{2} * g=\int_{\partial B} K_{\mathcal{N}, \alpha}(y-x) * \tilde{d} \sigma_{y} * f(y) * G^{-1} .
$$

Theorem 3.5 Let $B$ be as stated above, and $g=\left(\begin{array}{ll}g_{11} & g_{12} \\ g_{21} & g_{22}\end{array}\right)$ with entries belonging to $L^{p, 3}\left(R^{3}, \mathbb{H}(\mathbb{C})\right), 3<p<+\infty$. Find $w=\left(\begin{array}{l}w_{11} w_{12} \\ w_{21} w_{22}\end{array}\right) \in \operatorname{Mat}_{2 \times 2}(\mathbb{H}(\mathbb{C}))$ satisfying the following system:

$$
\left\{\begin{array}{l}
\mathcal{N}[w](x)=g(x), \quad x \in R^{3} \backslash \partial B, \\
w^{+}(\tau)=w^{-}(\tau) * G+f(\tau), \quad \tau \in \partial B, \\
w(x) \rightarrow 0, \quad \text { as } x \rightarrow \infty,
\end{array}\right.
$$

where $w^{ \pm}(\tau)=\lim _{x \in B^{ \pm}, x \rightarrow \tau} w(x), G=\left(\begin{array}{cc}G_{11} & 0 \\ 0 & G_{22}\end{array}\right)$ is quaternion constant matrix and its inverse

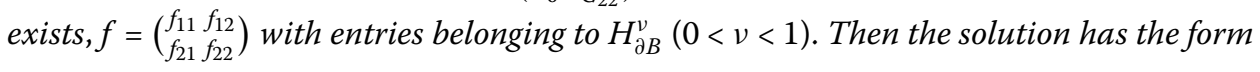

$$
w(x)=\Psi(x)+\left(T_{\mathcal{N}, \alpha}[g]\right)(x)
$$

in which $\mathcal{N}[\Psi](x)=0$ and

$$
\Psi(x)= \begin{cases}\int_{\partial B} \mathcal{K}_{\mathcal{N}, \alpha}(y-x) * \tilde{d} \sigma_{y} * \tilde{f}(y), & x \in B^{+}, \\ \int_{\partial B} \mathcal{K}_{\mathcal{N}, \alpha}(y-x) * \tilde{d} \sigma_{y} * \tilde{f}(y) * G^{-1}, & x \in B^{-},\end{cases}
$$

where $\tilde{f}=f+\left(T_{\mathcal{N}, \alpha}[g]\right) *(G-E)$. 
Proof By Theorem 3.3, we know

$$
\mathcal{N}[w]=\mathcal{N}\left[\Psi(x)+\left(T_{\mathcal{N}, \alpha}[g]\right)(x)\right]=g(x)
$$

The boundary condition (3.5) becomes

$$
\left(\Psi+T_{\mathcal{N}, \alpha}[g]\right)^{+}(\tau)=\left(\Psi+T_{\mathcal{N}, \alpha}[g]\right)^{-}(\tau) * G+f(\tau), \quad \tau \in \partial B .
$$

Again from Theorem 3.3, we know that $\left(T_{\mathcal{N}, \alpha}[g]\right)(x)$ has continuity in $\Omega \subset R^{3}$. Thus $\left(T_{\mathcal{N}, \alpha}[g]\right)^{+}=\left(T_{\mathcal{N}, \alpha}[g]\right)^{-}=T_{\mathcal{N}, \alpha}[g]$. Thus we can obtain

$$
\Psi^{+}(\tau)=\Psi^{-}(\tau) * G+\left(T_{\mathcal{N}, \alpha}[g]\right)(\tau) *(G-E)+f(\tau), \quad \tau \in \partial B
$$

Suppose $\tilde{f}=f+\left(T_{\mathcal{N}, \alpha}[g]\right) *(G-E)$, then (3.7) has the following form:

$$
\Psi^{+}(\tau)=\Psi^{-}(\tau) * G+\tilde{f}(\tau), \quad \tau \in \partial B
$$

Again from Theorem 3.4, the solutions which satisfy the system $\mathcal{N}[\Psi]=0$ and boundary condition (3.8) can be represented in the form

$$
\Psi(x)= \begin{cases}\int_{\partial B} \mathcal{K}_{\mathcal{N}, \alpha}(y-x) * \tilde{d} \sigma_{y} * \tilde{f}(y), & x \in B^{+} \\ \int_{\partial B} \mathcal{K}_{\mathcal{N}, \alpha}(y-x) * \tilde{d} \sigma_{y} * \tilde{f}(y) * G^{-1}, & x \in B^{-}\end{cases}
$$

\section{The Riemann boundary value problem related to the time-harmonic Maxwell equations}

4.1 The relevant definitions and operations

Let $\vec{E}, \vec{H}: \Omega \rightarrow \mathbb{C}^{3}$ be a pair of complex-valued vector fields. $\vec{E}=E_{1} i_{1}+E_{2} i_{2}+E_{3} i_{3}$, $\vec{H}=H_{1} i_{1}+H_{2} i_{2}+H_{3} i_{3}$, i.e. $E_{0}=0, H_{0}=0$. The following system:

$$
\begin{aligned}
& \operatorname{rot} \vec{H}=\sigma \vec{E}, \quad \operatorname{rot} \vec{E}=i \omega \mu \vec{H}, \\
& \operatorname{div} \vec{H}=0, \quad \operatorname{div} \vec{E}=0,
\end{aligned}
$$

is called the time-harmonic Maxwell equations. $(\vec{E}, \vec{H})$ is called a time-harmonic electromagnetic field, where $\sigma$ is a complex electrical conductivity and $\mu$ is a magnetic permeability. It is known that they satisfy the homogeneous Helmholtz equation

$$
\begin{aligned}
& \Delta \vec{E}+\lambda \vec{E}=0, \\
& \Delta \vec{H}+\lambda \vec{H}=0,
\end{aligned}
$$

where $\lambda=i \omega \mu \sigma \in \mathbb{C}, \alpha=\sqrt{\lambda}$. Set

$$
\mathcal{M}=\left(\begin{array}{cc}
\sigma & -\operatorname{rot} \\
\operatorname{rot} & -i \omega \mu
\end{array}\right)
$$


then Eq. (4.1) becomes

$$
\mathcal{M}\left[\left(\begin{array}{c}
\vec{E} \\
\vec{H}
\end{array}\right)\right]=0
$$

For $k \in Z^{+}$, set

$$
\hat{C}^{(k)}=\left\{\left(\begin{array}{l}
\vec{f} \\
\vec{g}
\end{array}\right) \in C^{(k)}\left(\Omega, \mathbb{C}^{3} \times \mathbb{C}^{3}\right) \mid \operatorname{div} \vec{f}=\operatorname{div} \vec{g}=0\right\}
$$

The operator

$$
\widehat{\mathcal{M}}=\mathcal{M}_{\mid \hat{C}^{(1)}}
$$

i.e. the restriction of $\mathcal{M}$ onto $\hat{C}^{(1)}$, will be termed the time-harmonic Maxwell operator. Then (4.1) and (4.2) become

$$
\widehat{\mathcal{M}}\left[\left(\begin{array}{c}
\vec{E} \\
\vec{H}
\end{array}\right)\right]=0
$$

where $\vec{E}, \vec{H} \in \hat{C}^{(1)}$

Let $\left(\begin{array}{ll}a & 0 \\ b & 0\end{array}\right) \in$ Mat $_{2 \times 2}$, which are identified naturally with columns $\left(\begin{array}{l}a \\ b\end{array}\right)$. We shall not distinguish them in this paper.

Let $\mathcal{N}: C^{(1)}\left(\Omega, \operatorname{Mat}_{2 \times 2}(\mathbb{H}(\mathbb{C}))\right) \rightarrow C^{(0)}\left(\Omega, \operatorname{Mat}_{2 \times 2}(\mathbb{H}(\mathbb{C}))\right)$. Set $\tilde{\mathcal{N}}=\mathcal{N}_{\mid \hat{C}^{(1)}}$, i.e. the restriction of $\mathcal{N}$ onto $\hat{C}^{(1)}$. Obviously, If $\left(\begin{array}{c}\vec{E} \\ \vec{H}\end{array}\right) \in \hat{C}^{(1)}$, then we have

$$
{ }^{\psi} D[\vec{E}]=-\operatorname{div} \vec{E}+\operatorname{rot} \vec{E}=\operatorname{rot} \vec{E}, \quad \psi D[\vec{H}]=-\operatorname{div} \vec{H}+\operatorname{rot} \vec{H}=\operatorname{rot} \vec{H}
$$

Therefore

$$
\widehat{\mathcal{M}}\left[\left(\begin{array}{c}
\vec{E} \\
\vec{H}
\end{array}\right)\right]=\left(\begin{array}{cc}
\sigma & -\operatorname{rot} \\
\operatorname{rot} & -i \omega \mu
\end{array}\right)\left(\begin{array}{c}
\vec{E} \\
\vec{H}
\end{array}\right)=\left(\begin{array}{cc}
\sigma & -{ }^{\psi} D \\
{ }^{\psi} D & -i \omega \mu
\end{array}\right)\left(\begin{array}{c}
\vec{E} \\
\vec{H}
\end{array}\right)=\tilde{\mathcal{N}}\left[\left(\begin{array}{c}
\vec{E} \\
\vec{H}
\end{array}\right)\right]
$$

That is, if $\left(\begin{array}{c}\vec{E} \\ \vec{H}\end{array}\right) \in \hat{C}^{(1)}$, then we have

$$
\widehat{\mathcal{M}}\left[\left(\begin{array}{c}
\vec{E} \\
\vec{H}
\end{array}\right)\right]=\tilde{\mathcal{N}}\left[\left(\begin{array}{c}
\vec{E} \\
\vec{H}
\end{array}\right)\right]
$$

Let $Q, R \in \operatorname{Mat}_{2 \times 2}(\mathbb{H}(\mathbb{C}))$, and $Q_{0}=\operatorname{Sc}(Q)=\left(\operatorname{Sc}\left(q_{m, n}\right)\right)_{1 \leq m, n \leq 2}, \quad \vec{Q}=\operatorname{Vec}(Q)=$ $\left(\vec{q}_{m, n}\right)_{1 \leq m, n \leq 2}$, set

$$
\langle\vec{Q}, \vec{R}\rangle=\left(\begin{array}{ll}
\left\langle\vec{q}_{11}, \vec{r}_{11}\right\rangle+\left\langle\vec{q}_{12}, \vec{r}_{21}\right\rangle & \left\langle\vec{q}_{11}, \vec{r}_{12}\right\rangle+\left\langle\vec{q}_{12}, \vec{r}_{22}\right\rangle \\
\left\langle\vec{q}_{21}, \vec{r}_{11}\right\rangle+\left\langle\vec{q}_{22}, \vec{r}_{21}\right\rangle & \left\langle\vec{q}_{21}, \vec{r}_{12}\right\rangle+\left\langle\vec{q}_{22}, \vec{r}_{22}\right\rangle
\end{array}\right),
$$




$$
[\vec{Q}, \vec{R}]=\left(\begin{array}{ll}
{\left[\vec{q}_{11}, \vec{r}_{11}\right]+\left[\vec{q}_{12}, \vec{r}_{21}\right]} & {\left[\vec{q}_{11}, \vec{r}_{12}\right]+\left[\vec{q}_{12}, \vec{r}_{22}\right]} \\
{\left[\vec{q}_{21}, \vec{r}_{11}\right]+\left[\vec{q}_{22}, \vec{r}_{21}\right]} & {\left[\vec{q}_{21}, \vec{r}_{12}\right]+\left[\vec{q}_{22}, \vec{r}_{22}\right]}
\end{array}\right)
$$

The time-harmonic Cauchy-Maxwell kernel is defined by

$$
\mathcal{K}_{\mathcal{M}, \alpha}(y-x)=\left(\left|\operatorname{grad} \theta_{\alpha}(y-x), \vec{n}_{y}\right\rangle,-\mathcal{U}_{\alpha}(y-x)\right)
$$

where $\theta_{\alpha}(y-x)$ be as stated, $\vec{n}_{y}$ is unit outward normal vector at $y \in \partial \Omega$, and

$$
\mathcal{U}_{\alpha}(y-x)=\left(\begin{array}{cc}
{\left[\operatorname{grad} \theta_{\alpha}(y-x), \vec{n}_{y}\right]} & i \omega \mu \theta_{\alpha}(y-x) \vec{n}_{y} \\
\sigma \theta_{\alpha}(y-x) \vec{n}_{y} & {\left[\operatorname{grad} \theta_{\alpha}(y-x), \vec{n}_{y}\right]}
\end{array}\right) .
$$

For any $\vec{g}=\left(\begin{array}{c}\vec{e} \\ \vec{h}\end{array}\right): \partial \Omega \rightarrow \mathbb{C}^{3} \times \mathbb{C}^{3}$, there is introduced the operation

$$
\mathcal{K}_{\mathcal{M}, \alpha}(y-x) \star \vec{g}(y)=\left\langle\operatorname{grad} \theta_{\alpha}(y-x), \vec{n}_{y}\right| \vec{g}(y)-\left[\mathcal{U}_{\alpha}(y-x), \vec{g}(y)\right],
$$

where

$$
\left[\mathcal{U}_{\alpha}(y-x), \vec{g}(y)\right]=\left(\begin{array}{c}
{\left[\left[\operatorname{grad} \theta_{\alpha}(y-x), \vec{n}_{y}\right], \vec{e}\right]+i \omega \mu \theta_{\alpha}(y-x)\left[\vec{n}_{y}, \vec{h}\right]} \\
\sigma \theta_{\alpha}(y-x)\left[\vec{n}_{y}, \vec{e}\right]+\left[\left[\operatorname{grad} \theta_{\alpha}(y-x), \vec{n}_{y}\right], \vec{h}\right]
\end{array}\right) .
$$

For any $V=\left(\begin{array}{ll}\vec{v}_{11} & \vec{v}_{12} \\ \vec{v}_{21} & \vec{v}_{22}\end{array}\right)$, there is introduced the operation

$$
V \diamond\left(\begin{array}{l}
\vec{e} \\
\vec{h}
\end{array}\right)=\left(\begin{array}{l}
\left\langle\vec{v}_{11}, \vec{e}\right\rangle+\left\langle\vec{v}_{12}, \vec{h}\right\rangle \\
\left\langle\vec{v}_{21}, \vec{e}\right\rangle+\left\langle\vec{v}_{22}, \vec{h}\right\rangle
\end{array}\right)
$$

Obviously, we have

$$
\left\langle\mathcal{U}_{\alpha}(y-x),\left(\begin{array}{l}
\vec{e} \\
\vec{h}
\end{array}\right)\right\rangle=\mathcal{U}_{\alpha}(y-x) \diamond\left(\begin{array}{l}
\vec{e} \\
\vec{h}
\end{array}\right) .
$$

Set

$$
\mathfrak{M}=\left\{\left(\begin{array}{l}
\vec{e} \\
\vec{h}
\end{array}\right): \partial \Omega \rightarrow \mathbb{C}^{3} \times \mathbb{C}^{3} \mid \int_{\partial \Omega} \mathcal{U}_{\alpha}(y-x) \diamond\left(\begin{array}{l}
\vec{e}(y) \\
\vec{h}(y)
\end{array}\right) d s_{y}=0, x \notin \partial \Omega\right\} .
$$

The integral

$$
K_{\mathcal{M}, \alpha}\left[\left(\begin{array}{l}
\vec{e} \\
\vec{h}
\end{array}\right)\right](x)=\int_{\partial \Omega} \mathcal{K}_{\mathcal{M}, \alpha}(y-x) \star\left(\begin{array}{l}
\vec{e}(y) \\
\vec{h}(y)
\end{array}\right) d s_{y}, \quad x \notin \partial \Omega,
$$

plays the role of an analogue of the Cauchy-Maxwell-type integral in the theory of timeharmonic electromagnetic fields.

\subsection{The Riemann boundary value problem related to time-harmonic Maxwell} equations

Lemma 4.1 $\operatorname{Set} \mathcal{K}_{\mathcal{M}, \alpha}^{0}(y-x)=\left\langle\operatorname{grad} \theta_{\alpha}(y-x), \vec{n}_{y}\right\rangle\left(\begin{array}{ll}1 & 0 \\ 0 & 1\end{array}\right)-\mathcal{U}_{\alpha}(y-x)$. Then

$$
\mathcal{K}_{\mathcal{N}, \alpha}(y-x) * \tilde{d} \sigma_{y}=\mathcal{K}_{\mathcal{M}, \alpha}^{0}(y-x) d s_{y}
$$


Proof By the definition of $\mathcal{K}_{\mathcal{N}, \alpha}(y-x)$, we have

$$
\begin{aligned}
\mathcal{K}_{\mathcal{N}, \alpha}(y-x) * \tilde{d} \sigma_{y} & =\frac{1}{2}\left(\begin{array}{cc}
\sigma^{-1} & \sigma^{-1} \\
\alpha^{-1} & -\alpha^{-1}
\end{array}\right) *\left(\begin{array}{cc}
\alpha \overline{\mathcal{K}}_{\psi, \alpha}(y-x) & -\sigma \overline{\mathcal{K}}_{\psi, \alpha}(y-x) \\
\alpha \mathcal{K}_{\psi, \alpha}(y-x & \sigma \mathcal{K}_{\psi, \alpha}(y-x)
\end{array}\right) *\left(\begin{array}{cc}
0 & -\vec{n}_{y} \\
\vec{n}_{y} & 0
\end{array}\right) d s_{y} \\
= & \frac{1}{2}\left(\begin{array}{cc}
\sigma^{-1} & \sigma^{-1} \\
\alpha^{-1} & -\alpha^{-1}
\end{array}\right) *\left(\begin{array}{cc}
\alpha^{\psi} D_{\alpha}\left[\theta_{\alpha}\right](y-x) & -\sigma^{\psi} D_{\alpha}\left[\theta_{\alpha}\right](y-x) \\
\alpha^{\psi} \bar{D}_{\alpha}\left[\theta_{\alpha}\right](y-x) & \sigma^{\psi} \bar{D}_{\alpha}\left[\theta_{\alpha}\right](y-x)
\end{array}\right) *\left(\begin{array}{cc}
0 & -\vec{n}_{y} \\
\vec{n}_{y} & 0
\end{array}\right) d s_{y} \\
= & \frac{1}{2}\left(\begin{array}{cc}
\sigma^{-1} & \sigma^{-1} \\
\alpha^{-1} & -\alpha^{-1}
\end{array}\right) *\left(\begin{array}{cc}
\alpha\left(\alpha \theta_{\alpha}+\operatorname{grad} \theta_{\alpha}\right) & -\sigma\left(\alpha \theta_{\alpha}+\operatorname{grad} \theta_{\alpha}\right) \\
\alpha\left(\alpha \theta_{\alpha}-\operatorname{grad} \theta_{\alpha}\right) & \sigma\left(\alpha \theta_{\alpha}-\operatorname{grad} \theta_{\alpha}\right)
\end{array}\right) *\left(\begin{array}{cc}
0 & -\vec{n}_{y} \\
\vec{n}_{y} & 0
\end{array}\right) d s_{y} \\
= & \frac{1}{2}\left(\begin{array}{cc}
\sigma^{-1} & \sigma^{-1} \\
\alpha^{-1} & -\alpha^{-1}
\end{array}\right) *\left(\begin{array}{cc}
-\sigma\left(\alpha \theta_{\alpha}+\operatorname{grad} \theta_{\alpha}\right) \vec{n}_{y} & -\alpha\left(\alpha \theta_{\alpha}+\operatorname{grad} \theta_{\alpha}\right) \vec{n}_{y} \\
\sigma\left(\alpha \theta_{\alpha}-\operatorname{grad} \theta_{\alpha}\right) \vec{n}_{y} & -\alpha\left(\alpha \theta_{\alpha}-\operatorname{grad} \theta_{\alpha}\right) \vec{n}_{y}
\end{array}\right) d s_{y} \\
= & \frac{1}{2}\left(\begin{array}{rr}
2\left\langle\operatorname{grad} \theta_{\alpha}, \vec{n}_{y}\right\rangle-2\left[\operatorname{grad} \theta_{\alpha}, \vec{n}_{y}\right] & -2 \sigma^{-1} \alpha^{2} \theta_{\alpha} \vec{n}_{y} \\
-2 \sigma \theta_{\alpha} \vec{n}_{y} & 2\left\langle\operatorname{grad} \theta_{\alpha}, \vec{n}_{y}\right\rangle-2\left[\operatorname{grad} \theta_{\alpha}, \vec{n}_{y}\right]
\end{array}\right) d s_{y} \\
= & \left(\begin{array}{rr}
\left\langle\operatorname{grad} \theta_{\alpha}, \vec{n}_{y}\right\rangle-\left[\operatorname{grad} \theta_{\alpha}, \vec{n}_{y}\right] & -\sigma^{-1} \alpha^{2} \theta_{\alpha} \vec{n}_{y} \\
-\sigma \theta_{\alpha} \vec{n}_{y} & \left\langle\operatorname{grad} \theta_{\alpha}, \vec{n}_{y}\right\rangle-\left[\operatorname{grad} \theta_{\alpha}, \vec{n}_{y}\right]
\end{array}\right) d s_{y} .
\end{aligned}
$$

By the definition of $\mathcal{K}_{\mathcal{M}, \alpha}^{0}$, we can obtain

$$
\begin{aligned}
& \mathcal{K}_{\mathcal{M}, \alpha}^{0}(y-x) d s_{y} \\
& =\left\{\left\langle\operatorname{grad} \theta_{\alpha}, \vec{n}_{y}\right\rangle\left(\begin{array}{ll}
1 & 0 \\
0 & 1
\end{array}\right)-\mathcal{U}_{\alpha}(y-x)\right\} d s_{y} \\
& =\left(\begin{array}{cc}
\left\langle\operatorname{grad} \theta_{\alpha}, \vec{n}_{y}\right\rangle-\left[\operatorname{grad} \theta_{\alpha}, \vec{n}_{y}\right] & -i \omega \mu \theta_{\alpha} \vec{n}_{y} \\
-\sigma \theta_{\alpha} \vec{n}_{y} & \left\langle\operatorname{grad} \theta_{\alpha}, \vec{n}_{y}\right\rangle-\left[\operatorname{grad} \theta_{\alpha}, \vec{n}_{y}\right]
\end{array}\right) d s_{y} \\
& =\left(\begin{array}{cc}
\left\langle\operatorname{grad} \theta_{\alpha}, \vec{n}_{y}\right\rangle-\left[\operatorname{grad} \theta_{\alpha}, \vec{n}_{y}\right] & -\sigma^{-1} \alpha^{2} \theta_{\alpha} \vec{n}_{y} \\
-\sigma \theta_{\alpha} \vec{n}_{y} & \left\langle\operatorname{grad} \theta_{\alpha}, \vec{n}_{y}\right\rangle-\left[\operatorname{grad} \theta_{\alpha}, \vec{n}_{y}\right]
\end{array}\right) d s_{y},
\end{aligned}
$$

where $\theta_{\alpha}=\theta_{\alpha}(y-x)$. Therefore $\mathcal{K}_{\mathcal{N}, \alpha}(y-x) * \tilde{d} \sigma_{y}=\mathcal{K}_{\mathcal{M}, \alpha}^{0}(y-x) d s_{y}$.

Lemma 4.2 If $\vec{f}=\left(\begin{array}{c}\vec{e} \\ \vec{h}\end{array}\right) \in \mathfrak{M}$. Then we have

$$
K_{\mathcal{M}, \alpha}\left[\left(\begin{array}{l}
\vec{e} \\
\vec{h}
\end{array}\right)\right](x)=K_{\mathcal{N}, \alpha}\left[\left(\begin{array}{l}
\vec{e} \\
\vec{h}
\end{array}\right)\right](x) .
$$

Proof By Lemma 4.1, we have

$$
\begin{aligned}
\mathcal{K}_{\mathcal{N}, \alpha}(y-x) * \tilde{d} \sigma_{y} * \vec{f}(y) \\
\quad=\mathcal{K}_{\mathcal{M}, \alpha}^{0}(y-x) * \vec{f}(y) d s_{y} \\
\quad=\left\{\left\langle\operatorname{grad} \theta_{\alpha}(y-x), \vec{n}_{y}\right\rangle\left(\begin{array}{ll}
1 & 0 \\
0 & 1
\end{array}\right)-\mathcal{U}_{\alpha}(y-x)\right\} * \vec{f}(y) d s_{y} \\
\quad=\left\{\left\langle\operatorname{grad} \theta_{\alpha}(y-x), \vec{n}_{y}\right| \vec{f}(y)-\left[\mathcal{U}_{\alpha}(y-x), \vec{f}(y)\right]+\left\langle\mathcal{U}_{\alpha}(y-x), \vec{f}(y)\right\rangle\right\} d s_{y}
\end{aligned}
$$




$$
=\left(\left\langle\mathcal{U}_{\alpha}(y-x), \vec{f}(y)\right\rangle+\mathcal{K}_{\mathcal{M}, \alpha}(y-x) \star \vec{f}(y)\right) d s_{y} .
$$

Therefore

$$
\begin{aligned}
K_{\mathcal{N}, \alpha}\left[\left(\begin{array}{l}
\vec{e} \\
\vec{h}
\end{array}\right)\right](x) & =\int_{\partial \Omega} \mathcal{K}_{\mathcal{N}, \alpha}(y-x) * \tilde{d} \sigma_{y} * \vec{f}(y) \\
& =\int_{\partial \Omega}\left(\left\langle\mathcal{U}_{\alpha}(y-x), \vec{f}(y)\right\rangle+\mathcal{K}_{\mathcal{M}, \alpha}(y-x) \star \vec{f}(y)\right) d s_{y} \\
& =\int_{\partial \Omega} \mathcal{K}_{\mathcal{M}, \alpha}(y-x) \star \vec{f}(y) d s_{y}+\int_{\partial \Omega} \mathcal{U}_{\alpha}(y-x) \diamond\left(\begin{array}{l}
\vec{e}(y) \\
\vec{h}(y)
\end{array}\right) d s_{y} \\
& =\int_{\partial \Omega} \mathcal{K}_{\mathcal{M}, \alpha}(y-x) \star \vec{f}(y) d s_{y}=K_{\mathcal{M}, \alpha}\left[\left(\begin{array}{l}
\vec{e} \\
\vec{h}
\end{array}\right)\right](x) .
\end{aligned}
$$

Lemma 4.3 If $\vec{f}(y)=\left(\begin{array}{l}\vec{e}(y) \\ \vec{h}(y)\end{array}\right) \in C^{(1)}\left(\Omega, \mathbb{C}^{3} \times \mathbb{C}^{3}\right)$ and $\vec{f}(y) \in \mathfrak{M}$. Then

$$
\int_{\partial B} \mathcal{K}_{\mathcal{M}, \alpha}(y-x) \star \vec{f}(y) d s_{y} \in \hat{C}^{(1)} .
$$

Proof By the definition, we have

$$
\begin{aligned}
\int_{\partial B} & \mathcal{K}_{\mathcal{M}, \alpha}(y-x) \star \vec{f}(y) d s_{y} \\
= & \int_{\partial B}\left\{\left\langle\operatorname{grad} \theta_{\alpha}, \vec{n}_{y}\right\rangle\left(\begin{array}{l}
\vec{e} \\
\vec{h}
\end{array}\right)-\left[\mathcal{U}_{\alpha}(y-x), \vec{f}(y)\right]\right\} d s_{y} \\
= & \left(\begin{array}{c}
\int_{\partial B}\left\{\left\langle\operatorname{grad} \theta_{\alpha}, \vec{n}_{y}\right\rangle \vec{e}-\left[\left[\operatorname{grad} \theta_{\alpha}, \vec{n}_{y}\right], \vec{e}\right]-i \omega \mu \theta_{\alpha}\left[\vec{n}_{y}, \vec{h}\right]\right\} d s_{y} \\
\int_{\partial B}\left\{\left\langle\operatorname{grad} \theta_{\alpha}, \vec{n}_{y}\right\rangle \vec{h}-\sigma \theta_{\alpha}\left[\vec{n}_{y}, \vec{e}\right]-\left[\left[\operatorname{grad} \theta_{\alpha}, \vec{n}_{y}\right], \vec{h}\right]\right\} d s_{y}
\end{array}\right),
\end{aligned}
$$

where $\theta_{\alpha}=\theta_{\alpha}(y-x)$.

(i) Since

$$
\begin{aligned}
& \operatorname{grad} \theta_{\alpha}=\left(\frac{\partial \theta_{\alpha}}{\partial x_{1}}, \frac{\partial \theta_{\alpha}}{\partial x_{2}}, \frac{\partial \theta_{\alpha}}{\partial x_{3}}\right), \quad \vec{n}_{y}=\left(y_{1}, y_{2}, y_{3}\right), \\
& \vec{e}=\left(e_{1}, e_{2}, e_{3}\right), \quad \vec{h}=\left(h_{1}, h_{2}, h_{3}\right),
\end{aligned}
$$

we can obtain

$$
\left\langle\operatorname{grad} \theta_{\alpha}, \vec{n}_{y}\right\rangle \vec{e}=\left(\frac{\partial \theta_{\alpha}}{\partial x_{1}} y_{1}+\frac{\partial \theta_{\alpha}}{\partial x_{2}} y_{2}+\frac{\partial \theta_{\alpha}}{\partial x_{3}} y_{3}\right)\left(e_{1}, e_{2}, e_{3}\right) .
$$

Therefore

$$
\begin{aligned}
\operatorname{div}\left\{\left\langle\operatorname{grad} \theta_{\alpha}, \vec{n}_{y}\right\rangle \vec{e}\right\} \\
=\frac{\partial\left(\frac{\partial \theta_{\alpha}}{\partial x_{1}} y_{1}+\frac{\partial \theta_{\alpha}}{\partial x_{2}} y_{2}+\frac{\partial \theta_{\alpha}}{\partial x_{3}} y_{3}\right)}{\partial x_{1}} e_{1}+\frac{\partial\left(\frac{\partial \theta_{\alpha}}{\partial x_{1}} y_{1}+\frac{\partial \theta_{\alpha}}{\partial x_{2}} y_{2}+\frac{\partial \theta_{\alpha}}{\partial x_{3}} y_{3}\right)}{\partial x_{2}} e_{2} \\
\quad+\frac{\partial\left(\frac{\partial \theta_{\alpha}}{\partial x_{1}} y_{1}+\frac{\partial \theta_{\alpha}}{\partial x_{2}} y_{2}+\frac{\partial \theta_{\alpha}}{\partial x_{3}} y_{3}\right)}{\partial x_{3}} e_{3}
\end{aligned}
$$




$$
\begin{aligned}
= & \frac{\partial^{2} \theta_{\alpha}}{\partial x_{1}^{2}} y_{1} e_{1}+\frac{\partial^{2} \theta_{\alpha}}{\partial x_{2}^{2}} y_{2} e_{2}+\frac{\partial^{2} \theta_{\alpha}}{\partial x_{3}^{2}} y_{3} e_{3}+\frac{\partial^{2} \theta_{\alpha}}{\partial x_{1} x_{2}}\left(y_{1} e_{2}+y_{2} e_{1}\right) \\
& +\frac{\partial^{2} \theta_{\alpha}}{\partial x_{1} x_{3}}\left(y_{1} e_{3}+y_{3} e_{1}\right)+\frac{\partial^{2} \theta_{\alpha}}{\partial x_{2} x_{3}}\left(y_{2} e_{3}+y_{3} e_{2}\right) .
\end{aligned}
$$

(ii) By the definition of the vector product, we have

$$
\begin{aligned}
{\left[\operatorname{grad} \theta_{\alpha}, \vec{n}_{y}\right]=} & \left(\frac{\partial \theta_{\alpha}}{\partial x_{2}} y_{3}-\frac{\partial \theta_{\alpha}}{\partial x_{3}} y_{2}\right) i_{1}+\left(\frac{\partial \theta_{\alpha}}{\partial x_{3}} y_{1}-\frac{\partial \theta_{\alpha}}{\partial x_{1}} y_{3}\right) i_{2} \\
& +\left(\frac{\partial \theta_{\alpha}}{\partial x_{1}} y_{2}-\frac{\partial \theta_{\alpha}}{\partial x_{2}} y_{1}\right) i_{3} .
\end{aligned}
$$

Thus

$$
\begin{aligned}
{\left[\left[\operatorname{grad} \theta_{\alpha}, \vec{n}_{y}\right], \vec{e}\right]=} & {\left[\left(\frac{\partial \theta_{\alpha}}{\partial x_{3}} y_{1}-\frac{\partial \theta_{\alpha}}{\partial x_{1}} y_{3}\right) e_{3}-\left(\frac{\partial \theta_{\alpha}}{\partial x_{1}} y_{2}-\frac{\partial \theta_{\alpha}}{\partial x_{2}} y_{1}\right) e_{2}\right] i_{1} } \\
& +\left[\left(\frac{\partial \theta_{\alpha}}{\partial x_{1}} y_{2}-\frac{\partial \theta_{\alpha}}{\partial x_{2}} y_{1}\right) e_{1}-\left(\frac{\partial \theta_{\alpha}}{\partial x_{2}} y_{3}-\frac{\partial \theta_{\alpha}}{\partial x_{3}} y_{2}\right) e_{3}\right] i_{2} \\
& +\left[\left(\frac{\partial \theta_{\alpha}}{\partial x_{2}} y_{3}-\frac{\partial \theta_{\alpha}}{\partial x_{3}} y_{2}\right) e_{2}-\left(\frac{\partial \theta_{\alpha}}{\partial x_{3}} y_{1}-\frac{\partial \theta_{\alpha}}{\partial x_{1}} y_{3}\right) e_{1}\right] i_{3} .
\end{aligned}
$$

Then

$$
\begin{aligned}
\operatorname{div}\{[ & {\left.\left.\left[\operatorname{grad} \theta_{\alpha}, \vec{n}_{y}\right], \vec{e}\right]\right\} } \\
= & -\frac{\partial^{2} \theta_{\alpha}}{\partial x_{1}^{2}}\left(y_{2} e_{2}+y_{3} e_{3}\right)-\frac{\partial^{2} \theta_{\alpha}}{\partial x_{2}^{2}}\left(y_{1} e_{1}+y_{3} e_{3}\right)-\frac{\partial^{2} \theta_{\alpha}}{\partial x_{3}^{2}}\left(y_{1} e_{1}+y_{2} e_{2}\right) \\
& +\frac{\partial^{2} \theta_{\alpha}}{\partial x_{1} x_{2}}\left(y_{1} e_{2}+y_{2} e_{1}\right)+\frac{\partial^{2} \theta_{\alpha}}{\partial x_{1} x_{3}}\left(y_{1} e_{3}+y_{3} e_{1}\right)+\frac{\partial^{2} \theta_{\alpha}}{\partial x_{2} x_{3}}\left(y_{2} e_{3}+y_{3} e_{2}\right) .
\end{aligned}
$$

(iii) By the definition of vector product, we have

$$
\left[\vec{n}_{y}, \vec{h}\right]=\left|\begin{array}{ccc}
i_{1} & i_{2} & i_{3} \\
y_{1} & y_{2} & y_{3} \\
h_{1} & h_{2} & h_{3}
\end{array}\right|=\left(y_{2} h_{3}-h_{2} y_{3}\right) i_{1}+\left(y_{3} h_{1}-h_{3} y_{1}\right) i_{2}+\left(y_{1} h_{2}-h_{1} y_{2}\right) i_{3} .
$$

Thus

$$
i \omega \mu \theta_{\alpha}\left[\vec{n}_{y}, \vec{h}\right]=\left(i \omega \mu \theta_{\alpha}\left(y_{2} h_{3}-h_{2} y_{3}\right), i \omega \mu \theta_{\alpha}\left(y_{3} h_{1}-h_{3} y_{1}\right), i \omega \mu \theta_{\alpha}\left(y_{1} h_{2}-h_{1} y_{2}\right)\right) .
$$

Then

$$
\operatorname{div}\left\{i \omega \mu \theta_{\alpha}\left[\vec{n}_{y}, \vec{h}\right]\right\}=i \omega \mu\left[\frac{\partial \theta_{\alpha}}{\partial x_{1}}\left(y_{2} h_{3}-h_{2} y_{3}\right)+\frac{\partial \theta_{\alpha}}{\partial x_{2}}\left(y_{3} h_{1}-h_{3} y_{1}\right)+\frac{\partial \theta_{\alpha}}{\partial x_{3}}\left(y_{1} h_{2}-h_{1} y_{2}\right)\right]
$$

Combining (i)-(iii), we have

$$
\begin{aligned}
& \operatorname{div}\left\{\left\langle\operatorname{grad} \theta_{\alpha}, \vec{n}_{y}\right\rangle \vec{e}-\left[\left[\operatorname{grad} \theta_{\alpha}, \vec{n}_{y}\right], \vec{e}\right]-i \omega \mu \theta_{\alpha}\left[\vec{n}_{y}, \vec{h}\right]\right\} \\
& \quad=\left(\frac{\partial^{2} \theta_{\alpha}}{\partial x_{1}^{2}}+\frac{\partial^{2} \theta_{\alpha}}{\partial x_{2}^{2}}+\frac{\partial^{2} \theta_{\alpha}}{\partial x_{3}^{2}}\right)\left(y_{1} e_{1}+y_{2} e_{2}+y_{3} e_{3}\right)-i \omega \mu\left[\frac{\partial \theta_{\alpha}}{\partial x_{1}}\left(y_{2} h_{3}-h_{2} y_{3}\right)\right.
\end{aligned}
$$




$$
\left.+\frac{\partial \theta_{\alpha}}{\partial x_{2}}\left(y_{3} h_{1}-h_{3} y_{1}\right)+\frac{\partial \theta_{\alpha}}{\partial x_{3}}\left(y_{1} h_{2}-h_{1} y_{2}\right)\right]
$$

Obviously, we have

$$
\frac{\partial^{2} \theta_{\alpha}}{\partial x_{1}^{2}}+\frac{\partial^{2} \theta_{\alpha}}{\partial x_{2}^{2}}+\frac{\partial^{2} \theta_{\alpha}}{\partial x_{3}^{2}}=\Delta \theta_{\alpha}=\Delta_{\alpha^{2}} \theta_{\alpha}-\alpha^{2} \theta_{\alpha}=-\alpha^{2} \theta_{\alpha}
$$

By the definition of vector product, we have

$$
\begin{aligned}
{\left[\operatorname{grad} \theta_{\alpha}, \vec{n}_{y}\right]=} & \left(\frac{\partial \theta_{\alpha}}{\partial x_{2}} y_{3}-\frac{\partial \theta_{\alpha}}{\partial x_{3}} y_{2}\right) i_{1}+\left(\frac{\partial \theta_{\alpha}}{\partial x_{3}} y_{1}-\frac{\partial \theta_{\alpha}}{\partial x_{1}} y_{3}\right) i_{2} \\
& +\left(\frac{\partial \theta_{\alpha}}{\partial x_{1}} y_{2}-\frac{\partial \theta_{\alpha}}{\partial x_{2}} y_{1}\right) i_{3} .
\end{aligned}
$$

Then

$$
\begin{aligned}
& \left\langle\left[\operatorname{grad} \theta_{\alpha}, \vec{n}_{y}\right], \vec{h}\right\rangle \\
& \quad=\left(\frac{\partial \theta_{\alpha}}{\partial x_{2}} y_{3}-\frac{\partial \theta_{\alpha}}{\partial x_{3}} y_{2}\right) h_{1}+\left(\frac{\partial \theta_{\alpha}}{\partial x_{3}} y_{1}-\frac{\partial \theta_{\alpha}}{\partial x_{1}} y_{3}\right) h_{2}+\left(\frac{\partial \theta_{\alpha}}{\partial x_{1}} y_{2}-\frac{\partial \theta_{\alpha}}{\partial x_{2}} y_{1}\right) h_{3} \\
& \quad=\frac{\partial \theta_{\alpha}}{\partial x_{1}}\left(y_{2} h_{3}-h_{2} y_{3}\right)+\frac{\partial \theta_{\alpha}}{\partial x_{2}}\left(y_{3} h_{1}-h_{3} y_{1}\right)+\frac{\partial \theta_{\alpha}}{\partial x_{3}}\left(y_{1} h_{2}-h_{1} y_{2}\right) .
\end{aligned}
$$

Since $\vec{f}(y)=\left(\begin{array}{c}\vec{e}(y) \\ \vec{h}(y)\end{array}\right) \in \mathfrak{M}$, we have $\int_{\partial B} \mathcal{U}_{\alpha}(y-x) \diamond\left(\begin{array}{c}\vec{e}(y) \\ \vec{h}(y)\end{array}\right) d s_{y}=0$. In addition, by the definition of $\diamond$, we have

$$
\begin{aligned}
\int_{\partial B} \mathcal{U}_{\alpha}(y-x) \diamond\left(\begin{array}{c}
\vec{e}(y) \\
\vec{h}(y)
\end{array}\right) d s_{y} \\
=\int_{\partial B}\left(\begin{array}{c}
\left\langle\left[\operatorname{grad} \theta_{\alpha}, \vec{n}_{y}\right], \vec{e}\right\rangle+i \omega \mu \theta_{\alpha}\left\langle\vec{n}_{y}, \vec{h}\right\rangle \\
\sigma \theta_{\alpha}\left\langle\vec{n}_{y}, \vec{e}\right\rangle+\left\langle\left[\operatorname{grad} \theta_{\alpha}, \vec{n}_{y}\right], \vec{h}\right\rangle
\end{array}\right) d s_{y} \\
=\left(\begin{array}{c}
\int_{\partial B}\left\{\left\langle\left[\operatorname{grad} \theta_{\alpha}, \vec{n}_{y}\right], \vec{e}\right\rangle+i \omega \mu \theta_{\alpha}\left\langle\vec{n}_{y}, \vec{h}\right\rangle\right\} d s_{y} \\
\int_{\partial B}\left\{\sigma \theta_{\alpha}\left\langle\vec{n}_{y}, \vec{e}\right\rangle+\left\langle\left[\operatorname{grad} \theta_{\alpha}, \vec{n}_{y}\right], \vec{h}\right\rangle\right\} d s_{y}
\end{array}\right) .
\end{aligned}
$$

Therefore

$$
\int_{\partial B}\left\{\sigma \theta_{\alpha}\left\langle\vec{n}_{y}, \vec{e}\right\rangle+\left\langle\left[\operatorname{grad} \theta_{\alpha}(y-x), \vec{n}_{y}\right], \vec{h}\right\rangle\right\} d s_{y}=0 .
$$

By (4.3)-(4.6), we have

$$
\begin{aligned}
\operatorname{div} & \int_{\partial B}\left\{\left\langle\operatorname{grad} \theta_{\alpha}, \vec{n}_{y}\right\rangle \vec{e}-\left[\left[\operatorname{grad} \theta_{\alpha}, \vec{n}_{y}\right], \vec{e}\right]-i \omega \mu \theta_{\alpha}\left[\vec{n}_{y}, \vec{h}\right]\right\} d s_{y} \\
= & \int_{\partial B} \operatorname{div}\left\{\left\langle\operatorname{grad} \theta_{\alpha}, \vec{n}_{y}\right\rangle \vec{e}-\left[\left[\operatorname{grad} \theta_{\alpha}, \vec{n}_{y}\right], \vec{e}\right]-i \omega \mu \theta_{\alpha}\left[\vec{n}_{y}, \vec{h}\right]\right\} d s_{y} \\
= & \int_{\partial B}\left\{-\alpha^{2} \theta_{\alpha}\left\langle\vec{n}_{y}, \vec{e}\right\rangle-i \omega \mu\left\langle\left[\operatorname{grad} \theta_{\alpha}, \vec{n}_{y}\right], \vec{h}\right\rangle\right\} d s_{y} \\
= & -i \omega \mu \int_{\partial B}\left\{\sigma \theta_{\alpha}\left\langle\vec{n}_{y}, \vec{e}\right\rangle+\left\langle\left[\operatorname{grad} \theta_{\alpha}, \vec{n}_{y}\right], \vec{h}\right\rangle\right\} d s_{y}=0 .
\end{aligned}
$$


Similarly, we have

$$
\operatorname{div} \int_{\partial B}\left\{\left\langle\operatorname{grad} \theta_{\alpha}, \vec{n}_{y}\right\rangle \vec{h}-\sigma \theta_{\alpha}\left[\vec{n}_{y}, \vec{e}\right]-\left[\left[\operatorname{grad} \theta_{\alpha}, \vec{n}_{y}\right], \vec{h}\right]\right\} d s_{y}=0
$$

Therefore

$$
\int_{\partial B} \mathcal{K}_{\mathcal{M}, \alpha}(y-x) \star \vec{f}(y) d s_{y} \in \hat{C}^{(1)} .
$$

Theorem 4.1 Let $B$ be as stated above. Find $u(x)=\left(\begin{array}{l}\vec{u}_{11} \\ \vec{u}_{21}\end{array}\right) \in \hat{C}^{(1)}$ satisfying the following system:

$$
\left\{\begin{array}{l}
\mathcal{M}[u]=0, \quad x \in R^{3} \backslash \partial B, \\
u^{+}(\tau)=u^{-}(\tau) * G+\vec{f}(\tau), \quad \tau \in \partial B, \\
u(x) \rightarrow 0, \quad \text { as } x \rightarrow \infty
\end{array}\right.
$$

where $u^{ \pm}(\tau)=\lim _{x \in B^{ \pm}, x \rightarrow \tau} u(x), G=\left(\begin{array}{c}G_{11} \\ 0\end{array}\right)$ is a quaternion constant matrix and its inverse exists, $\vec{f}=\left(\begin{array}{c}\vec{f}_{11} \\ \vec{f}_{21}\end{array}\right) \in \mathfrak{M}$ with entries belonging to $H_{\partial B}^{v}(0<v<1)$. Then the solution can be expressed as

$$
u(x)= \begin{cases}\int_{\partial B} \mathcal{K}_{\mathcal{M}, \alpha}(y-x) \star \vec{f}(y) d s_{y}, & x \in B^{+}, \\ \int_{\partial B} \mathcal{K}_{\mathcal{M}, \alpha}(y-x) \star \vec{f}(y) d s_{y} * G^{-1}, & x \in B^{-} .\end{cases}
$$

Proof Since $u(x) \in \hat{C}^{(1)}$, we have $N(u)=\tilde{N}(u)=\widehat{\mathcal{M}}(u)=\mathcal{M}(u)=0$. By Theorem 3.4, then the solution can be expressed as

$$
u(x)= \begin{cases}\int_{\partial B} \mathcal{K}_{\mathcal{N}, \alpha}(y-x) * \widetilde{d} \sigma_{y} * \vec{f}(y), & x \in B^{+} \\ \int_{\partial B} \mathcal{K}_{\mathcal{N}, \alpha}(y-x) * \tilde{d} \sigma_{y} * \vec{f}(y) * G^{-1}, & x \in B^{-}\end{cases}
$$

Since $\vec{f}=\left(\begin{array}{l}\vec{f}_{11} \\ \vec{f}_{21}\end{array}\right) \in \mathfrak{M}$, by Lemma 4.2 and Lemma 4.3, the solution can be expressed as

$$
u(x)= \begin{cases}\int_{\partial B} \mathcal{K}_{\mathcal{M}, \alpha}(y-x) \star \vec{f}(y) d s_{y}, & x \in B^{+}, \\ \int_{\partial B} \mathcal{K}_{\mathcal{M}, \alpha}(y-x) \star \vec{f}(y) d s_{y} * G^{-1}, & x \in B^{-},\end{cases}
$$

and $u(x) \in \hat{C}^{(1)}$.

\section{Acknowledgements}

The authors are thankful to the anonymous referees for reading the manuscript and giving fruitful comments and suggestions.

\section{Funding}

This work was supported by the National Natural Science Foundation of China (No. 12071479, No. 11401162, No. 11871191), the Natural Science Foundation of Hebei Province (No. A2020205008), the Key Foundation of Hebei Normal University (No. L2021Z01), and the Outstanding Innovative Talents Cultivation Funded Programs 2019 of Renmin Univertity of China. 


\section{Competing interests}

The authors declare that they have no competing interests.

\section{Authors' contributions}

All the authors contributed equally in this research. All authors read and approved the final manuscript.

\section{Publisher's Note}

Springer Nature remains neutral with regard to jurisdictional claims in published maps and institutional affiliations.

Received: 24 July 2020 Accepted: 25 August 2021 Published online: 13 September 2021

\section{References}

1. Vekua, N.: Generalized Analytic Functions. Pergamon, Oxford (1962)

2. Hile, G.N.: Elliptic systems in the plane with first order terms and constant coefficients. Commun. Partial Differ. Equ. 3, 949-977 (1978)

3. Gilbert, R.P., Hou, Z.Y., Meng, X.W.: Vekua theory in higher dimensional complex spaces: the $\pi$-operator in $C^{n}$. Complex Var. Theory Appl. 1, 99-105 (1993)

4. Du, J.Y., Xu, N.: Boundary behavior of Cauchy-type integrals in Clifford analysis. Acta Math. Sci. 29B(1), $210-224$ (2009)

5. Du, J.Y., Xu, N.: On boundary behavior of the Cauchy type integrals with values in a universal Clifford algebra. Adv. Appl. Clifford Algebras 21(1), 49-87 (2011)

6. Gu, L.F., Du, J.Y., Cai, D.H.: A kind of Riemann boundary value problems for pseudo-harmonic functions in Clifford analysis. Complex Var. Elliptic Equ. 3, 412-426 (2014)

7. Yang, P.W.: The Dirichlet boundary value problems for some quaternion functions of higher order on the polydisc. Sci. Sin. 41(6), 85-496 (2011)

8. Huang, S., Qiao, Y.Y., Wen, G.C.: Real and Complex Clifford Analysis. Springer, New York (2006)

9. Qiao, Y.Y., Wang, L.P., Yang, G.M.: A kind of boundary value problem for inhomogeneous partial differential system. J. Inequal. Appl. 180, 1-13 (2016)

10. Taira, K.: Degenerate elliptic boundary value problems with asymptotically linear nonlinearity. Rend. Circ. Mat. Palermo 60, 283-308 (2011)

11. Wang, L.P., Qiao, Y.Y., Yang, H.J.: Some properties of the Teodorescu operator related to the $\alpha$-Dirac operator. Appl. Anal. 93(11), 2413-2425 (2014)

12. Wang, L.P., Wen, G.C.: Boundary value problems for two types of degenerate elliptic systems in $R^{4}$. Appl. Math. J. Chin. Univ. 31(4), 469-480 (2016)

13. Wang, L.P., Xu, Z.L., Qiao, Y.Y.: The mixed boundary value problem for the inhomogeneous Cimmino system. Bound. Value Probl. 13, 1-16 (2015)

14. Wang, L.P., Yang, H.J., Xie, Y.H., Qiao, Y.Y.: Riemann boundary value problem for a kind of weighted Dirac operator in quaternion analysis. Sci. Sin., Math. 45(11), 1919-1930 (2015) (in Chinese)

15. Yang, P., Wang, L.P., Gao, L.: Some properties and applications of the Teodorescu operator associated to the Helmholtz equation. J. Inequal. Appl. 264, 1-19 (2017)

16. Wang, L.P.: Some properties of a kind of generalized Teodorescu operator in Clifford analysis. J. Inequal. Appl. 102, 1-11 (2016)

17. Mcintosh, A., Mitrea, M.: Clifford algebras and Maxwell's equations in Lipschitz domains. Math. Methods Appl. Sci. 22(18), 1599-1620 (1999)

18. Schneider, B., Shapiro, M.: Some properties of the Cauchy-type integral for the time-harmonic Maxwell equations. Integral Equ. Oper. Theory 44(1), 93-126 (2002)

19. Schneider, B., Shapiro, M.: Some properties of the Cauchy-type integral for the time-harmonic relativistic Dirac equation. Math. Methods Appl. Sci. 25(16-18), 1441-1463 (2002)

20. Kravchenko, V.V., Shapiro, M.V.: Quaternionic time-harmonic Maxwell operator. J. Phys. A, Math. Gen. 28(17), 5017-5031 (1994)

21. Kravchenko, V.V.: Applied quaternionic analysis: Maxwell's system and Dirac's equation. In: Functional-Analytic and Complex Methods, Their Interactions and Applications to Partial Differential Equations - Proceedings of the International Graz Workshop, pp. 143-160 (2001)

22. Kravchenko, V.V., Shapiro, M.V.: Integral Representations for Spatial Models of Mathematical Physics. Pitman Research Notes in Mathematics Series. Longman, Harlow (1996)

23. Russell, D.L.: The Dirichlet-Neumann boundary problem associated with the control theory of Maxwell's equations. In: IEEE Conference on Decision and Control (1983)

24. Yang, P.W., Yang, S., Li, M.L.: An initial-boundary value problem for the Maxwell equations. J. Differ. Equ. 249(12), 3003-3023 (2010)

25. Colton, D., Kress, R.: Integral Equation Methods in Scattering Theory \|| Chap. 4: Boundary-Value Problems for the Time-Harmonic Maxwell Equations and the Vector Helmholtz Equation. Wiley, New York (2013)

26. Abreu-Blaya, R., Ávila-Ávila, R., Bory-Reyes, J., Rodríguez-Dagnino, R.M.: Cauchy representation formulas for Maxwell equations in 3-dimensional domains with fractal boundaries. Bull. Braz. Math. Soc. 46(4), 681-700 (2015) 